This document was prepared in conjunction with work accomplished under Contract No. DE-AC09-96SR18500 with the U. S. Department of Energy.

\title{
DISCLAIMER
}

This report was prepared as an account of work sponsored by an agency of the United States Government. Neither the United States Government nor any agency thereof, nor any of their employees, makes any warranty, express or implied, or assumes any legal liability or responsibility for the accuracy, completeness, or usefulness of any information, apparatus, product or process disclosed, or represents that its use would not infringe privately owned rights. Reference herein to any specific commercial product, process or service by trade name, trademark, manufacturer, or otherwise does not necessarily constitute or imply its endorsement, recommendation, or favoring by the United States Government or any agency thereof. The views and opinions of authors expressed herein do not necessarily state or reflect those of the United States Government or any agency thereof.

This report has been reproduced directly from the best available copy.

Available for sale to the public, in paper, from: U.S. Department of Commerce, National Technical Information Service, 5285 Port Royal Road, Springfield, VA 22161, phone: (800) 553-6847, fax: (703) 605-6900

email: orders@ntis.fedworld.gov

online ordering: http://www.ntis.gov/help/index.asp

Available electronically at http://www.osti.gov/bridge

Available for a processing fee to U.S. Department of Energy and its contractors, in paper, from: U.S. Department of Energy, Office of Scientific and Technical Information, P.O. Box 62, Oak Ridge, TN 37831-0062,

phone: (865)576-8401,

fax: (865)576-5728

email: $\underline{\text { reports@ adonis.osti.gov }}$ 
This page was intentionally left blank 
WSRC-TR-2002-00232

Key Words: Aluminosilicate

Zeolite

Deposition

Uranium

\section{Concentrate Interaction Testing}

S.W. Rosencrance

T.B. Edwards

D.P. Healy

E.J. Coleman

Publication Date: June 28, 2002 


\section{TABLE OF CONTENTS}

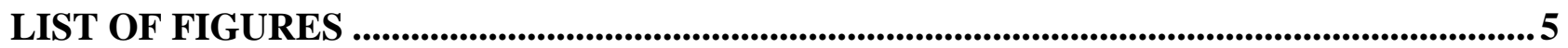

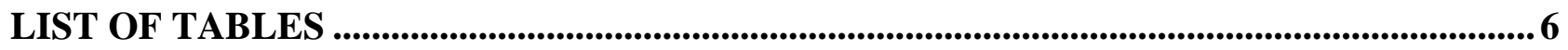

EXECUTIVE SUMMARY ...............................................................................................................................

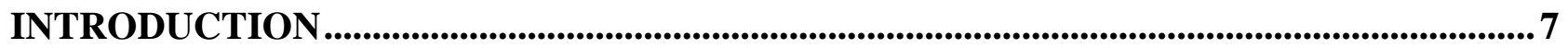

OBJECTIVES ......................................................................................................................................................... 8

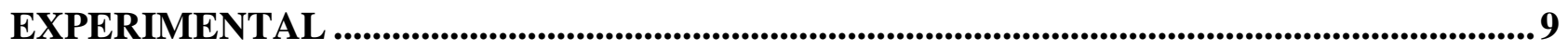

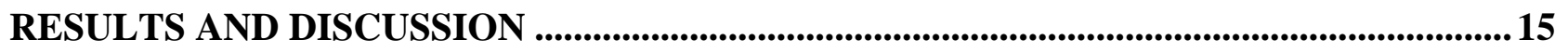

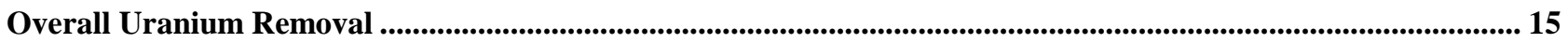

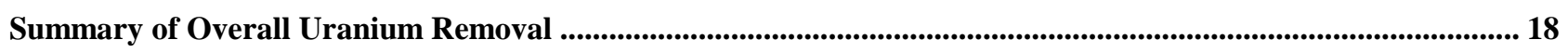

The Statistical Model to Assist in Explaining Uranium Removal ......................................................................................... 18

Discussion of Statistically Signifigant Terms .................................................................................................................... 22

The Role of Al and Si Stoichiometry on Overall Uranium Removal ............................................................................... 24

Error! Bookmark not defined.Overall Application of Results to Plant Operation ............................................................ 28

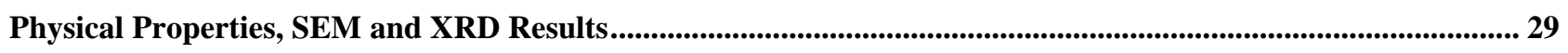

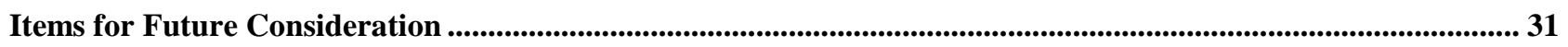

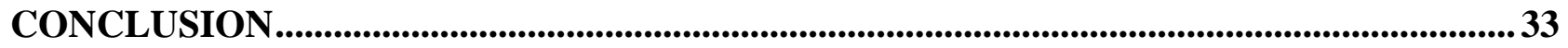

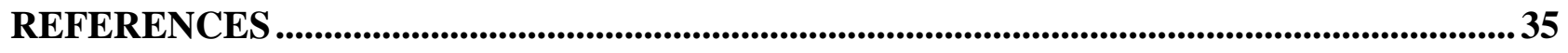

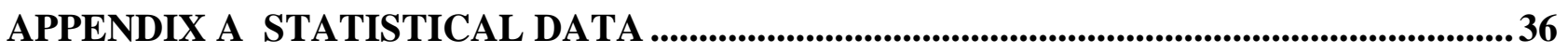


Rosencrance, et al.

WSRC-TR-2002-00232

Page 4 of 51

Exhibit A1 Plots of \% U Removed versus the Experimental Factors.............................................................................. 37

Exhibit A2 Results of Fitting the Full Quadratic Model to the \% U Removed Data .................................................. 38

Exhibit A2 Results of Fitting the Full Model to the \% U Removed Data (continued)............................................... 39

Exhibit A3 Results of Fitting the Reduced Model to the \% U Removed Data............................................................40

APPENDIX B FACTOR COMBINATIONS LEADING TO POSITIVE EFFECT OF SIGMA

TERMS ON URANIUM REMOVAL ......................................................................................42

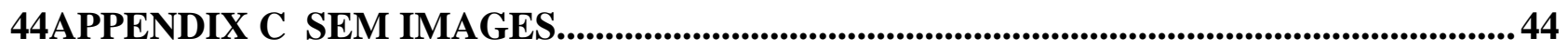




\section{List of Figures}

Figure 1 Experimentally Determined Vs. Predicted \% U Removed........................................ 19

Figure 2 Interaction Plots for Reduced Model Fitted to \%U Removed .................................21

Figure 3 Average Effect of Sigma on \%U Removed .......................................................................26

Figure 4 Effect of Individual Combinations of Factor Levels on \%U Removed...................... 28 
Rosencrance, et al.

WSRC-TR-2002-00232

Page 6 of 51

\section{LIST OF TABLES}

Table 1 Factor Levels and their Absolute Values.................................................................... 13

Table 24 Runs of Primary Experimental Design.................................................................. 14

Table 38 Run Secondary Experimental Design................................................................ 14

Table $4 \%$ U Removed versus Coded Factor Levels .................................................................... 17

Table $5 \%$ U Removed for Eight Stoichiometric Runs (in Coded Factor Levels)...................25 


\section{Executive Summary}

Testing was performed to define technical issues that may impact long-term viability of the segregation strategy for processing and storing evaporator concentrates. The primary concern is increased potential for nuclear criticality from uranium accumulation. This statistically designed testing examines the fate of uranium when $\mathrm{Al}$ and $\mathrm{Si}$ rich concentrates are either purposefully or inadvertently combined.

Uranium was removed from solution in all tests. The uranium removal was well described by a statistical model. The physical properties of the resulting solids were also evaluated. The solids were brittle and could be dispersed by introducing shear.

In the majority of cases uranium removal was decreased with increasing initial concentration (supersaturation) of silicate and/or aluminate. This trend is hypothesized to be the result of a dominant impact of silicate and/or aluminate species on the activity and ultimately solubility of uranium. This proposed effect on uranium solubility may explain previous experimental observations of higher soluble uranium fractions than anticipated.

Under some conditions, which are statistically signifigant, the opposite trend is observed and it is inferred that another removal process is dominant. Namely, that uranium removal increases with increasing concentrations of silicate and/or aluminate and is dominated by a process other than the previously discussed impact of silicate and aluminate on uranium solubility. One potential explanation for this trend would be "scrubbing" of uranium during the formation of aluminosilicate solid phases.

In general these tests demonstrate that uranium removal is expected when aluminate and silicate rich concentrates are combined if the initial solution contains $20 \mathrm{ppm}$ or more of uranium. Increasing the initial uranium concentration would be expected to increase the uranium removal. Similarly it would be expected that uranium removal would decrease as the initial uranium concentration is decreased. Thus if uranium containing concentrates that are rich in silicate and aluminate are combined uranium solids formation processes are possible. 


\section{Introduction}

The 242-16H High Level Waste Evaporator processes radioactive waste from the feed tank (Tank $43 \mathrm{H})$, concentrates the waste, and discharges to the concentrate receipt tank (Tank 38H). During this processing the waste typically concentrates by 30-40 vol. \%. However, during processing of Defense Waste Processing Facility Recycle stream, the concentration approaches 90 vol. \%. Included in the DWPF stream is dissolved and entrained silica. The silicon reacts with soluble aluminate ion to form an insoluble sodium aluminosilicate with a general chemical formula of $\mathrm{Na}_{8} \mathrm{Al}_{6} \mathrm{Si}_{6} \mathrm{O}_{24}\left(\mathrm{NO}_{3}\right)_{2}$. This material has been found in the Gravity Drain Line ${ }^{1}$ and inside the Evaporator Pot. ${ }^{2}$

Previous testing ${ }^{3}$ showed the formation of aluminosilicate was rapid under evaporator operating conditions. Testing has also been performed on the solid precipitate under a wide range of chemical conditions. ${ }^{4}$ Testing has been performed to assist in evaluating the deposition of aluminosilicates on stainless steel substrates. ${ }^{5,6}$

One operational scenario for consideration both from safety and operational perspectives is commonly referred to as segregation. The strategy employed in segregation is to isolate silicon containing streams and aluminum containing streams. By not allowing the two reactants to mix the resulting formation and potential deposition problems affiliated with aluminosilicates are mitigated. Information is needed in order to evaluate the long-term viability of this segregation based operational strategy. Namely, an understanding of the mixing, intentional or accidental, of these segregated and highly concentrated streams is needed.

\section{Objectives}

The current testing was performed in order to assist in defining technical issues that surround the segregation strategy. This testing is focussed on segregated concentrates that mixed and allowed to interact. The partition of uranium between solid and liquid phases was evaluated. Specifically, this removal of uranium serves as an indicator for the amount of uranium precipitated as well as the effect of sigma (supersaturation with respect to $\mathrm{Si}$ and $\mathrm{Al}$ ) on the uranium removal from solution. 
Rosencrance, et al.

WSRC-TR-2002-00232

Page 9 of 51

The removal observed in this work is a simulation of when enriched concentrates of silicon and aluminum that were formed during segregation either purposefully or inadvertently interact. If uranium solids are not formed then segregation viability in the long-term is clearly favorable. If uranium is removed from solution then one must consider if the amount and type of solids removed are of concern from a criticality perspective.

This concentrate interaction testing was a direct result of considering the possibility of an accelerated removal of uranium from solution via an efficient interaction of uranium with various aluminosilicate precursor phases. These interactions, if significant, could lead to a means of accentuating uranium removal in liquors that contain appreciable aluminate and/or silicate.

This specific concern was founded on a mass balance of uranium, aluminum, and silicon around the fouled $2 \mathrm{H}$ evaporator as prepared by E.J. Lahoda. Specifically, it was postulated that a higher fraction of uranium was retained in the accumulated mass of the fouled $2 \mathrm{H}$-evaporator relative to retention of $\mathrm{Si}$ and $\mathrm{Al}$ species. As a result of this mass balance it has been postulated that the primary mechanism for uranium removal could be scrubbing of uranium via aluminosilicate precursors.

In addition to evaluating uranium removal the solids that were formed were also qualitatively evaluated for potentially problematic cementation/fusion characteristics. This testing was in response to a technical task request ${ }^{7}$ and examined the uranium partition and solids properties as a function of temperature, sigma, sludge, concentration factor, and time.

\section{Experimental}

These concentrate interaction tests were conducted in typical waste simulants. ${ }^{8}$ These simulated waste concentrates contained sodium hydroxide, sodium nitrate, sodium nitrite, silicate, and aluminate in various ratios. The tests were performed on a scale of 300-ml. Samples were prepared with varying concentration factors, sigma, sludge, times, and temperatures. 
Rosencrance, et al.

WSRC-TR-2002-00232

Page 10 of 51

Silicon rich and aluminum rich solutions were prepared to various levels of sigma (supersaturation). Sigma is defined as the ratio of the difference between an initial concentration of a given species and the equilibrium concentration of the species of interest to the equilibrium concentration of the species of interest. Thus a value of zero would be equal to a saturated system. Values of sigma greater than 0 represent increasing levels of sigma. Uranium was introduced at $20 \mathrm{ppm}^{9}$. These solutions were then mixed to initiate the aluminosilicate formation and placed in a double shelled container system prior to being placed in an oven at a specified temperature for prescribed times.

A series of 32 runs were performed per a statistically-developed, experimental design. The primary response variable was the partition of uranium between the liquid and solid phases as a function of the experimental variables. A secondary evaluation focussed qualitatively on the physical properties of the resulting solids and the extent of their fusion/cementation.

In order to form simulated concentrate feed solutions that demonstrated well-characterized reaction profiles the silicon rich and aluminum rich solutions were not mixed until the experiment was initiated. The above solutions were formulated in a manner such that when equal volumes of the two solutions were mixed the resulting solution had the desired final concentrations of aluminum, silicon, hydroxide, sodium, nitrate, and nitrite. An exact balance of solution composition was not possible because of small amounts of counter-ions present from introduction of silicon and aluminum. All solutions were created compositionally such that the hydroxide, sodium, silicon, and aluminum levels were constant as desired for a given combination of concentration factor and supersaturation. The nitrate/nitrite ratio was variable in some cases in order to meet the constancy for the other species. There is not a mathematical solution to maintain all constant in this simple system given the small amounts of sodium included in the sodium silicate and the nitrate in the aluminum nitrate that were used as reagents. In all cases a stoichimetric excess of nitrate and nitrite was present for formation of the aluminosilicate structures of interest.

In order to obtain the final volume of 300-ml and the appropriate final concentration factor and sigma, mixtures were formed using equal volumes (150-ml) of both the silicon and aluminum containing stock solutions. These solutions were placed in a 500-ml PolyMethylPentane (PMP) 
Rosencrance, et al.

WSRC-TR-2002-00232

Page 11 of 51

bottle and swirled for at least 30 seconds. The PMP bottles had previously been demonstrated to be stable at temperatures of $90^{\circ} \mathrm{C}$ for extended periods of time when containing caustic solutions such as those employed in this testing. Furthermore, the bottles are rated in the literature to be used under these types of conditions. The neck of the PMP bottle had a small pinhole drilled to alleviate any small amounts of pressure that accumulated. The hole was small enough that evaporation was negligible (less than $1 \%$ ).

Immediately following the mixture of the silicon and aluminum solutions, the simulated sludge was added as necessary. The targeted level was a simulated sludge carryover of $0.8 \mathrm{wt} . \%$. To accomplish this, 7.8 grams of the simulated sludge was added and swirled for 30 seconds. Following sludge addition, uranium was added to each solution. The amount of uranium calculated for introduction was $20 \mathrm{ppm}$ of depleted uranium. The uranium source was uranyl nitrate and was prepared in a $0.1 \mathrm{M}$ nitric acid solution by adding 0.51 grams and diluting to $200-\mathrm{ml}$ total volume. A 5-ml aliquot of this stock solution was added to the 300-ml silicon/aluminum combined solution for each run. Following the addition, swirling of the contents ensued for 30 seconds. At this point two 5-ml samples were taken from the bottle corresponding to each experimental run. One sample was submitted to ADS for determination via ICP-MS of the initial uranium concentration and the second was archived for future use.

The 500-ml PMP bottle containing the experimental solution was then sealed and placed inside of a 1-liter glass bottle as a secondary container. The lid was taped per Health Protection (HP) personnel instruction and the sample placed in the appropriate oven for the specified amount of time.

Temperature was measured daily and found to always be $\pm 2{ }^{\circ} \mathrm{C}$ of the target value.

After the specified time the samples were removed from the oven. A video was made of the bottle immediately following removal and then two 5-ml samples were taken. Both samples were filtered using a 0.45-micron nylon cup filter. The first sample was sent to ADS for ICP-MS determination of uranium. The second sample was archived in the event of the need for submission of another sample at a later time. Two samples of the solids were also taken (submitted to ADS for XRD and SEM at our discretion). The second sample was again archived for potential future use. 
Rosencrance, et al.

WSRC-TR-2002-00232

Page 12 of 51

The requested variables to be examined were the extent of concentration, sigma, sludge presence, time, and temperature as shown in Table 1. The observables of interest were uranium partition between solid and liquid and the characteristics of the resulting solids. Given the number of variables or factors, it was decided that use of a statistically-designed experiment would be beneficial. The resulting design is a variant of a fractional factorial with centerpoints. The centerpoints will aid in the detection of curvilinear effects. Main effects and two-way interactions are not confounded. Replicates were included for model robustness. The midpoints for time and sigma were fully centered but the resulting effect on the designs statistical power to resolve is extremely small. Table 1 supplies detailed information on the levels of the various factors that were explored.

The design consisted of two parts. The primary part consisted of 24 runs and was used to evaluate the detailed effect of the five factors in systems with equi-molar initial silicon and aluminum. The secondary design consisted of 8 runs that were at ratios of aluminum/silicon of 5:1 and 1:5 to yield insight into effects of stoichiometry if any. These runs were all placed at the midpoint to provide a glimpse into the importance of the starting ratio. The two experimental designs are shown in Tables 2 and 3. Note that the three levels are denoted as 1, 0 and -1 and correspond to the actual levels given already in Table 1. 


\section{- Concentration Factor (1X, 1.3X and 1.6X)}

-1X- $\mathrm{Na} \approx 6 \mathrm{M}, \mathrm{OH} \approx 4 \mathrm{M}$, Nitrate $\approx 1 \mathrm{M}$

$\bullet 1.3 \mathrm{X}-\mathrm{Na} \approx 7.8 \mathrm{M}, \mathrm{OH} \approx 5.2 \mathrm{M}$, Nitrate $\approx 1.3 \mathrm{M}$

-1.6X- Na $\approx 9.6 \mathrm{M}, \mathrm{OH} \approx 6.4 \mathrm{M}$ Nitrate $\approx 1.6 \mathrm{M}$

-Supersaturation $(\sigma=0, \sigma=25$, and $\sigma=125)$

$\cdot \sigma=0$ is saturation

- $\sigma$ is with respect to $\mathrm{Si}_{\text {eq }}$ at $1: 1 \mathrm{~A} \mathrm{~d} / \mathrm{Si}$ ratio

- $\sigma$ is an initial target and actual will be calculate fromfinal data

- Sludge

-Simulated sludge was received fromBarnes et. Al in SRTC

-Per K. Gilbreath and W. Wilmarth $0.8 \mathrm{wt} \%$ was added using a $16 \mathrm{wt} \%$ simulant

-Temperature (40C, 50C, and 60C)

-Range of temperatures is pertinent to tanks/storage of concentrates

-Range allows investigation into possible temperature dependant modes of incorporation

-Midpoint is needed given curvilinear model requirements

- Time ( 3 days, 4 weeks, and 8 weeks)

$\bullet 8$ weeks should be equilibriumfor all samples

-Dynamics or uraniumpartition could be evaluated

Table 1 Factors of Design and Their Absolute Compositional Targets 


\begin{tabular}{|c|c|c|c|c|}
\hline $\begin{array}{c}\text { Saturation } \\
\frac{1}{0} \\
\frac{1}{1} \\
\frac{-1}{0} \\
\frac{1}{0} \\
\frac{0}{-1} \\
\frac{-1}{1} \\
\frac{-1}{-1} \\
\frac{-1}{0} \\
\frac{0}{1} \\
\frac{-1}{-1} \\
\frac{-1}{1} \\
\frac{0}{-1} \\
\frac{-1}{1} \\
1\end{array}$ & $\begin{array}{c}\text { Temperature } \\
\frac{1}{0} \\
\frac{-1}{-1} \\
\frac{0}{0} \\
\frac{1}{0} \\
\frac{1}{1} \\
\frac{1}{0} \\
\frac{0}{1} \\
\underline{0} \\
\frac{1}{-1} \\
\frac{1}{-1} \\
\frac{1}{1} \\
\frac{-1}{-1} \\
\frac{-1}{-1}\end{array}$ & $\begin{array}{c}\text { Con. Factor } \\
\frac{-1}{0} \\
\frac{-1}{-1} \\
\frac{-1}{0} \\
\frac{1}{0} \\
\frac{-1}{1} \\
\frac{1}{0} \\
\frac{0}{1} \\
\frac{0}{-1} \\
\frac{1}{1} \\
\frac{-1}{-1} \\
\frac{-1}{0} \\
\frac{-1}{1} \\
\frac{1}{1} \\
1\end{array}$ & $\begin{array}{l}\text { Time } \\
\frac{0}{0} \\
\frac{1}{1} \\
\frac{-1}{0} \\
\frac{1}{1} \\
\frac{0}{1} \\
\frac{1}{1} \\
\frac{0}{0} \\
\frac{-1}{0} \\
\frac{1}{-1} \\
\frac{-1}{-1} \\
\frac{-1}{-1} \\
\frac{-1}{0} \\
\frac{1}{1} \\
\frac{-1}{1}\end{array}$ & 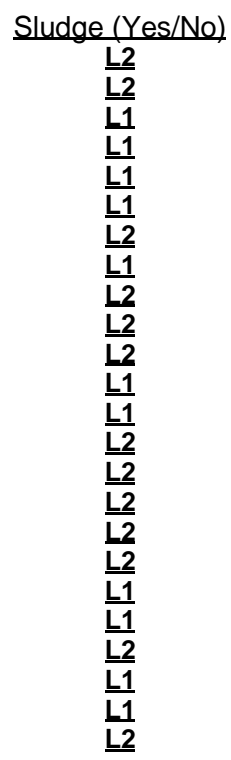 \\
\hline
\end{tabular}

Table 24 Run Primary Experimental Design

\begin{tabular}{|c|c|c|c|c|c|}
\hline Saturation & Temperature & $\underline{\text { Con. Factor }}$ & $\underline{\underline{\text { Time }}}$ & $\underline{\underline{\text { Sludge }}}$ & $\underline{\underline{\text { Al/Si Ratio }}}$ \\
\cline { 2 - 5 } & 0 & 0 & 0 & $\mathrm{Y}$ & $5: 1$ \\
0 & 0 & 0 & 0 & $\mathrm{Y}$ & $5: 1$ \\
0 & 0 & 0 & 0 & $\mathrm{Y}$ & $1: 5$ \\
0 & 0 & 0 & 0 & $\mathrm{Y}$ & $1: 5$ \\
0 & 0 & 0 & 0 & $\mathrm{~N}$ & $5: 1$ \\
0 & 0 & 0 & 0 & $\mathrm{~N}$ & $5: 1$ \\
0 & 0 & 0 & 0 & $\mathrm{~N}$ & $1: 5$ \\
0 & 0 & 0 & 0 & $\mathrm{~N}$ & $1: 5$ \\
\hline
\end{tabular}

Table $3 \quad 8$ Run Secondary Experimental Design 


\section{Results and Discussion}

\section{Overall Uranium Removal}

The targeted initial uranium concentration in each test solution was 20ppm. A homogeneous stock solution of uranium was prepared and $5 \mathrm{ml}$ added to each of the 32 experimental runs (24 in the primary experimental matrix and 8 in the secondary experimental matrix). The uranium was measured by ICP-MS following an acid dissolution. Due to a lengthy outage of the ICP-MS a decision was made to run all initial and final samples in batch aliquot mode to expedite turnaround time and maintain program milestones.

Initial attempts by ADS to collect an aliquot of the sample and perform the dissolution proved to yield extremely variable results. This variability was likely the result of formation of aluminosilicate phases in the sample bottles and this issue was likely accentuated during the several weeks that the ICP-MS was under repair. The accompanying heterogeneity of the sample made analysis of an aliquot unrepresentative and necessitated dissolution of the entire sample bottle. At this point five of the initial solutions were measured again by performing the dissolution on the entire bottle of archived samples in order to establish that the amount of uranium introduced was reproducible for each run. This initial analysis also allowed for a verification of the amount of uranium initially present in each of the experimental runs.

These five measurements performed on samples taken from bottles that were fully dissolved were 23.1, 24.7, 22.0, 23.1, and 21.3ppm. The mean of these five measurements is $22.8 \mathrm{ppm}$ with an associated $95 \%$ confidence interval (uncertainty for the mean assuming normality) of $\pm 1.6 \mathrm{ppm}$. These results clearly demonstrate that the desired amount of uranium was reproducibly introduced into the initial samples.

The original concentrations determined by the aliquot method for these same five samples were 15.8, 17.6, 16.5, 18.3, and 17.6ppm respectively. It became clear that the aliquot method appeared to be consistently lower than when the entire bottle underwent dissolution. An observation was made that the difference of the uranium concentrations for the aliquot method as compared to the full bottle 
Rosencrance, et al.

WSRC-TR-2002-00232

Page 16 of 51

dissolution method appeared to be constant at about $20 \%$ less for the aliquot method. Realizing that the final samples from the end of each experimental run could be experiencing the same measurement bias led the research team to also resubmit three of the final experimental samples whose concentrations of uranium were suspiciously low.

Interestingly, the results for the three final samples that were rerun also demonstrated this apparently constant experimental bias of about 20\%. When statistically reviewed, the ICP-MS data for the aliquot method average for the 8 full bottle dissolution samples is $21.2 \% \pm 2.7 \%$ with $95 \%$ confidence. The tightness of this interval strongly suggests a systematic experimental error associated with the aliquot analysis method.

The initial focus of these tests is to determine if uranium is removed from solution and associated with the solids. In order to make the most conservative determination of this, a comparison of the worst case should be made. Realizing that the initial level of uranium was $22.8 \mathrm{ppm}$ with a lower confidence limit of $21.2 \mathrm{ppm}$ then the upper limit of the final uranium concentrations would need to be less than this value for the full bottle dissolution method. For the three final samples that underwent the full dissolution the values were 10.4, 8.6, and 3.3ppm all of which are well below the required statistical threshold which is associated with a statistically significant removal of uranium from solution. However, the other 21 final samples (from the primary experimental design matrix of 24 runs) are from the aliquot method that is known to be biased low.

In order for the 21 samples analyzed using the aliquot method to demonstrate removal of uranium from solution their final upper confidence limit when corrected for the previously demonstrated systematic bias must be less than $21.2 \mathrm{ppm}$ also. In other words, final uranium concentrations from the aliquot method must be less than the quotient of $21.2 \mathrm{ppm}$ and the bias factor of 1.212 or a value of $17.5 \mathrm{ppm}$ in this most rigid and conservative interpretation, a large number of final samples analyzed by the aliquot method demonstrate final uranium concentrations of less than $17.5 \mathrm{ppm}$ (removal of greater than 23\%). Thus it can be safely concluded that a significant (based upon a worst case approach) amount of uranium was removed from solution during many of the experimental runs of these tests. To be exact 18 of the 24 primary experimental design tests 
Rosencrance, et al.

WSRC-TR-2002-00232

Page 17 of 51

demonstrated uranium removal from solution greater than the necessary $23 \%$ with final uranium removal for each experimental run given in Table 4.

$\begin{array}{cccccc}\text { Sigma } & \text { Conc } & \text { Temp } & \text { Time } & \text { Present } & \text { Removed } \\ 1 & -1 & 1 & 0 & \mathrm{Y} & 28 \\ 0 & 0 & 0 & 0 & \mathrm{Y} & 31 \\ 1 & -1 & -1 & 1 & \mathrm{n} & 18 \\ -1 & -1 & -1 & -1 & \mathrm{n} & 18 \\ 0 & 0 & 0 & 0 & \mathrm{n} & 23 \\ 1 & 1 & 1 & 1 & \mathrm{n} & 27 \\ 0 & 0 & 0 & 0 & \mathrm{Y} & 28 \\ -1 & -1 & 1 & 1 & \mathrm{n} & 17 \\ -1 & 1 & 1 & 1 & \mathrm{Y} & 54 \\ 1 & 0 & 1 & 1 & \mathrm{Y} & 32 \\ -1 & 0 & 0 & 0 & \mathrm{Y} & 27 \\ -1 & 1 & 1 & -1 & \mathrm{n} & 62 \\ 0 & 0 & 0 & 0 & \mathrm{n} & 20 \\ 0 & -1 & 0 & 1 & \mathrm{Y} & 25 \\ 1 & 1 & 1 & -1 & \mathrm{Y} & 34 \\ -1 & 1 & -1 & -1 & \mathrm{Y} & 28 \\ -1 & -1 & 1 & -1 & \mathrm{Y} & 27 \\ 1 & -1 & -1 & -1 & \mathrm{Y} & 25 \\ 1 & -1 & 1 & -1 & \mathrm{n} & 17 \\ 0 & 0 & 1 & 0 & \mathrm{n} & 23 \\ -1 & -1 & -1 & 1 & \mathrm{Y} & 27 \\ -1 & 1 & -1 & 1 & \mathrm{n} & 86 \\ 1 & 1 & -1 & -1 & \mathrm{n} & 30 \\ 1 & 1 & -1 & 1 & \mathrm{Y} & 33\end{array}$

\section{Table $4 \quad \%$ U Removed versus Coded Factor Levels}

The observed uranium removal spanned the range from $17 \%$ on the low side to $86 \%$ on the upperend. An interesting attribute of this sample grouping is that the greatest uranium removal was observed for the case where the system was not saturated with respect to aluminosilicate formation. This begins to suggest that one should consider the possibility that the presence of $\mathrm{Si}$ and/or $\mathrm{Al}$ 
Rosencrance, et al.

WSRC-TR-2002-00232

Page 18 of 51

impacts the activity coefficient and ultimately could decrease the solubility of uranium in these types of chemical matrices.

\section{Summary of Overall Uranium Removal}

For the level of uranium concentration initially introduced into these tests $(20 \mathrm{ppm})$ the data reveal that in all cases uranium is removed from solution and transferred to a solid phase. Furthermore, the vast majority of these tests $(75 \%)$ demonstrate a statistically significant removal or uranium from solution at a confidence level of $95 \%$. At slightly lower levels of confidence all samples demonstrated uranium solids formation.

As discussed earlier in this manuscript removal of uranium from solution is a necessary but not sufficient condition for criticality concerns. Further evaluation would be necessary to determine if this removal is an issue with regard to the viability of segregation in the longer-term.

\section{The Statistical Model to Assist in Explaining Uranium Removal}

The statistical design utilized a set of 24 design points that were selected using the D-optimality routine in the "Design of Experiments" platform of JMP Version 3.2.6 from SAS Institute, Inc. This design related the uranium removal from solution as the response of interest to the experimental factors given by the equation shown below:

$$
\begin{aligned}
\text { Response } & =\beta_{0}+\beta_{1} \cdot \text { Sigma }+\beta_{2} \cdot \text { Conc }+\beta_{3} \cdot \text { Sigma } \cdot \text { Conc }+\beta_{4} \cdot \text { Temp }+\beta_{5} \cdot \text { Sigma } \cdot \text { Temp } \\
& +\beta_{6} \cdot \text { Conc } \cdot \text { Temp }+\beta_{7} \cdot \text { Time }+\beta_{8} \cdot \text { Sigma } \cdot \text { Time }+\beta_{9} \cdot \text { Conc } \cdot \text { Time } \\
& +\beta_{10} \cdot \text { Temp } \cdot \text { Time }+\beta_{11} \cdot \text { Sludge }+\beta_{12} \cdot \text { Sigma } \cdot \text { Sludge }+\beta_{13} \cdot \text { Conc } \cdot \text { Sludge } \\
& +\beta_{14} \cdot \text { Temp } \cdot \text { Sludge }+\beta_{15} \cdot \text { Time } \cdot \text { Sludge }+\beta_{16} \cdot \text { Sigma }^{2}+\beta_{17} \cdot \text { Conc }^{2}+\beta_{18} \cdot \text { Temp }^{2} \\
& +\beta_{19} \cdot \text { Time }^{2}+\varepsilon
\end{aligned}
$$

where the $\beta$ 's are unknown parameters that are to be estimated using the experimental data and $\varepsilon$ represents a random error term that is assumed to have a mean of zero and a constant (but unknown) variance. Table 4 provides the results (expressed as \% U removed) from these experimental runs. 
Rosencrance, et al.

WSRC-TR-2002-00232

Page 19 of 51

The data in this table were analyzed using JMP Version 4.0.5. Exhibit A1 in Appendix A provides plots of the \%U removed versus each of the factors in Table 4. The results of using JMP to fit the data to the model given in the equation above are provided in Exhibit A2 in Appendix A. A "lack of fit" test is provided which indicates that "lack of fit" for this model is not statistically significant at the 5\% level. A table showing the correlations among the estimated terms of the model is also provided. Ideally, all of the off-diagonal terms should be zero. In this table, the largest correlation (in absolute value) is around 0.10 for the main effects and the two-way interactions indicating little correlation among these estimates. Higher correlations are seen among the squared terms and between squared terms and the main effects and two-way interactions. The largest (absolute) correlation is 0.585 .

The results in Exhibit A2 suggest that some of the interaction terms and quadratic terms in the model are not statistically significant (at the 5\% significance level). This is indicated by a value of "Prob $>|t|$ " in the "Parameter Estimates" section of the exhibit that is greater than 0.05 . The insignificant interactions include sigma·temperature, temperature concentration factor, and time-sludge. To facilitate the removal of insignificant terms from the model, JMP's stepwise regression routine was used to select a "best fitting" model. The results from this effort were further refined by excluding interactions terms in the model that were statistically insignificant at the 5\% level. The final results (for this reduced model) are provided in Exhibit A3 of Appendix A.

The resulting model demonstrates an $\mathrm{R}^{2}$ greater than $98 \%$ with no significant lack of fit. Figure 1 shows a plot of the experimentally determined $\%$ uranium removal versus the predicted $\%$ uranium removal using the reduced model. Also, shown in this plot is the $95 \%$ confidence interval for the expected \% uranium removal for the model. 


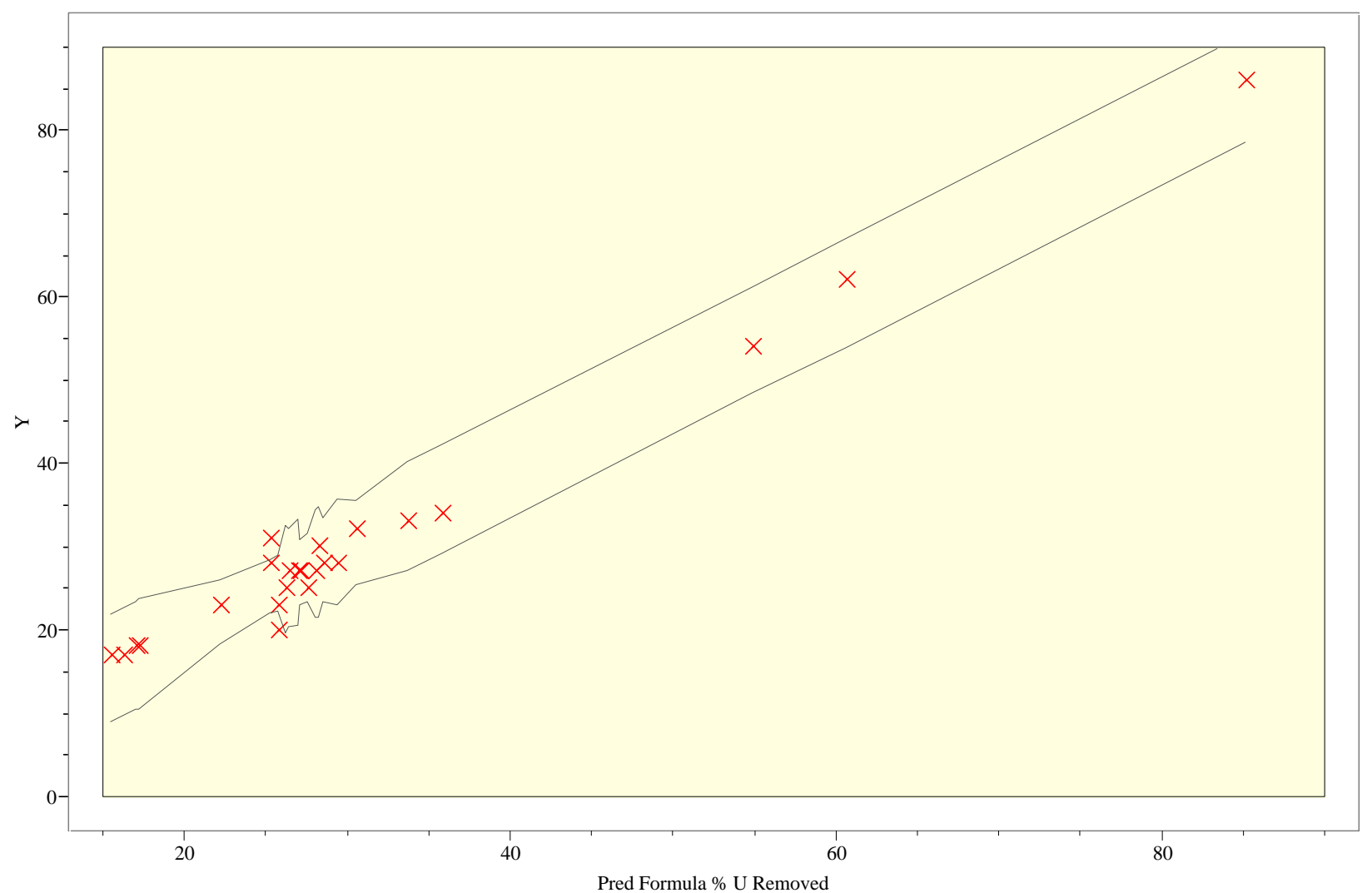

Figure 1 Experimentally Determined vs. Predicted \% U Removed

Note that all of the interactions appearing in the reduced model are statistically significant at the 5\% level and that all of the main effects remain in the model regardless of their statistical significance. All main effects remain in the model because all of the factors are involved in one or more statistically significant interactions. It is a customary practice to include in the model the main effect for each factor that is involved in significant interactions.

A closer look at the pair-wise interactions among the five experimental factors is provided in Figure 2. The interactions that are statistically significant are those whose two plotted lines are not parallel. Thus, from Figure 2, one sees significant interactions between sigma and sludge, sigma and concentration factor, temperature and time, sigma and sludge, concentration factor and sludge, and temperature and sludge. Looking back at the interactions shown as being significant in Exhibit A3 confirms these observations. 


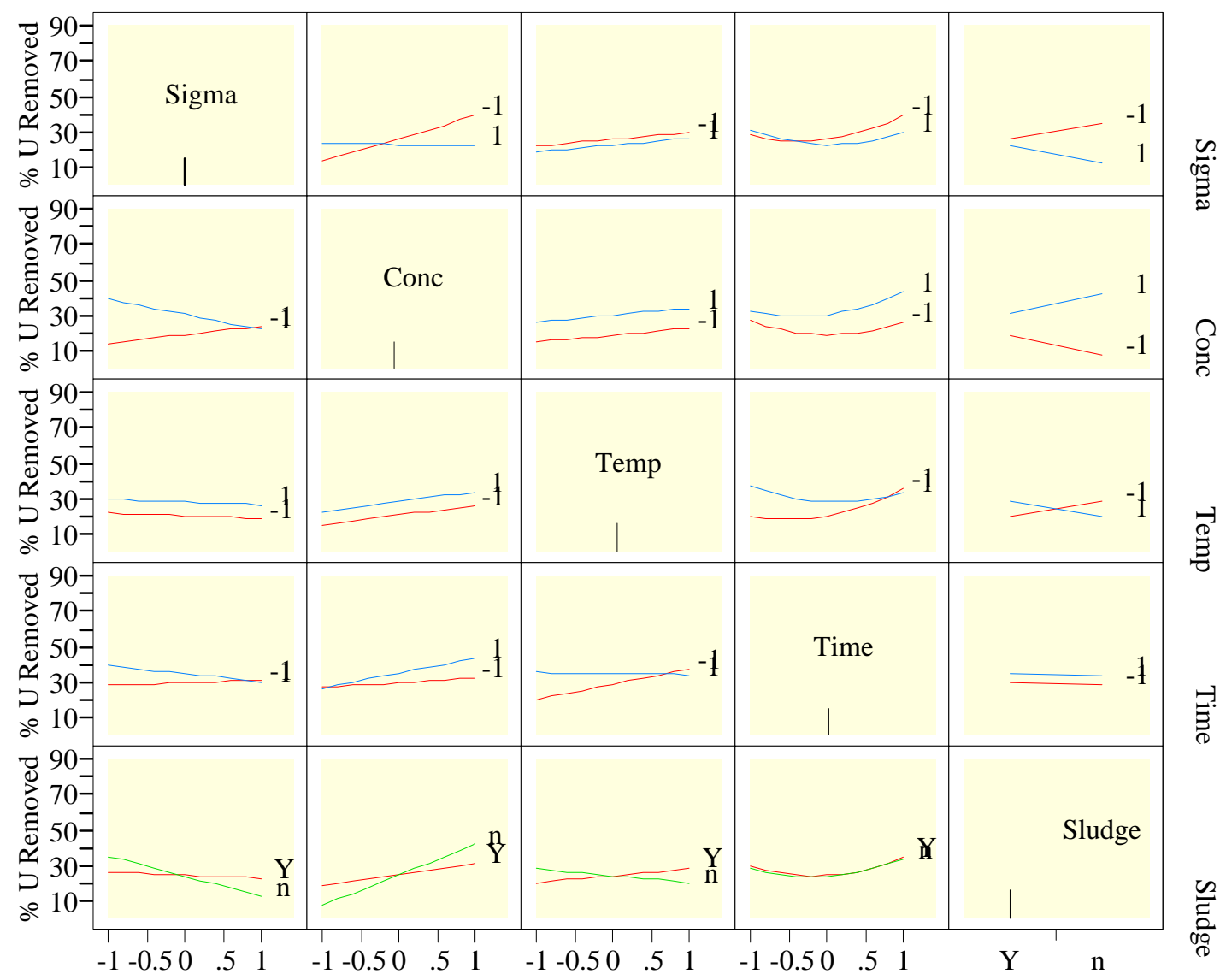

Figure 2 Interaction Plots for Reduced Model Fitted to \% U Removed

The first order main effects of sigma, concentration factor, and time are important at a 5\% level. The factor of time is also statistically pertinent when considered as a quadratic effect. Specifically, the model predicts that increasing sigma decreases uranium removal and that increasing concentration factor and increasing time both increase uranium removal. Physical interpretation of any of the model coefficients involves speculation and to further understand the physical pertinence of any of the model coefficients further work would be necessary. The explanations suggested are only meant to stimulate thought based on limited information. It is envisioned that much of the remaining aluminosilicate program underway this fiscal year will collectively work together to provide a more thorough understanding of these highly complex phenomena. 
Rosencrance, et al.

WSRC-TR-2002-00232

Page 22 of 51

\section{Discussion of Statistically Significant Terms}

One possible explanation for the role of sigma in the model is that the presence of $\mathrm{Si}$ and/or $\mathrm{Al}$ impacts the activity coefficient and ultimately could decrease the solubility of uranium in these types of chemical matrices. In other words, the presence of $\mathrm{Si}$ and/or $\mathrm{Al}$ (higher sigma) alters the activity of uranium in the simulated evaporator liquor. This alteration of the solubility is such that the impact on the amount of uranium removed from solution decreases with increased sigma because the solubility of the uranium has been increased.

The primary effect of concentration factor suggested by the model is that increased concentration factor leads to increased uranium removal. This effect is potentially due to the increased ionic strengths associated with increased concentration factor. It is known that compression of double layer and reduction of repulsive barriers occur under the shielding conditions of elevated ionic strength. This condition could possibly facilitate uranium removal from solution, again likely suggesting a heterogeneous removal mechanism.

The primary effect of time suggested by the model is that increased reaction time leads to increased uranium removal. This effect is statistically significant both as a linear and a quadratic contributor to the model. It is important to note that the quadratic term is partially confounded with other quadratic, linear, and interaction terms. However, these results strongly implicate the role of reaction time in the $\%$ of uranium removed from solution. From a physical perspective this finding is not surprising when one considers the apparent importance of the kinetic mismatch between aluminosilicate and uranium solid formation processes. It has been previously observed in SRTC by Pierce and Peterson that formation of uranium solids during an aluminosilicate synthesis is favored by increasing time due to the relatively slow kinetics of uranium solids formation relative to the kinetics typically associated with the formation of aluminosilicate solid phases.

For the sigma $\cdot$ concentration factor interaction, as concentration factor is taken from its low to high levels, the \% U removed tends to decrease slightly when sigma is at its high level but it tends to increase when sigma is at its low level. In other words, uranium removal from solution is enhanced 
Rosencrance, et al.

WSRC-TR-2002-00232

Page 23 of 51

when sigma and concentration factors are at opposite levels. This potentially supports the thought previously expressed that the kinetics of uranium removal processes and the kinetics of aluminosilicate formation processes are inherently mismatched in this type of batch testing and that repulsive interactions are reduced at increased concentration factors.

For the temperature - time interaction, as time is taken from its low to high levels, the \% $\mathrm{U}$ removed tends to decrease slightly when temperature is at its high level but it tends to increase when temperature is at its low level. Again, uranium removal is enhanced when these two factors are at opposite levels. In other words, short time and high temperature have a similar effect as long time and lower temperature. This again suggests an optimum synchronization for the aluminosilicate formation and the uranium solids formation. Specifically, if both time and temperature are low then the reaction to form aluminosilicate could be slow and limiting relative to the uranium solids formation process. While in the opposite case if both are high then the aluminosilicate process is driven to occur prior to the uranium formation process and removal of uranium from solution is reduced. Only when they are opposites would they provide the necessary balance of aluminosilicate rate to synchronize with the uranium formation process.

For the concentration factor - sludge interaction, as concentration factor is taken from its low to high levels, the \% U removed tends to increase slightly when sludge is present (sludge = "Y") but it tends to increase even more dramatically when sludge is not present. This result can also be restated that when sludge is present and the system has significantly higher particulate surface area available for uranium removal that the efficiency gained in the absence of sludge by reducing repulsive barriers at increased concentration factors is not nearly as pertinent. In other words, the higher particulate seed surface provided by the sludge could provide some increased efficiency relative to the no sludge case thus the impact of increased concentration factor on reducing repulsion and facilitating uranium removal is lessened.

For the temperature sludge interaction, as temperature is taken from its low to high levels, the \% $\mathrm{U}$ removed tends to increase slightly when sludge is present (sludge = "Y") but it tends to decrease slightly when sludge is not present. This particular interaction term, although significant in the 
Rosencrance, et al.

WSRC-TR-2002-00232

Page 24 of 51

statistical model, is difficult to physically interpret. One potential explanation could lie in the relative temperature dependant solubility of the simulated sludge and the aluminosilicates. If the sludge solubility was less sensitive to temperature then the possibility exists that by increasing temperature one could observe in the case with a absence of sludge the system actually dissolves increasing fractions of the aluminosilicates which could potentially free previously removed uranium. This explanation is complicated by the fact that increasing solution temperature also favors a more rapid transformation to the less soluble aluminosilicate phases also which would contrast the projected results of this interaction term. At best, the physical understanding of this particular term in the model is difficult.

For the sigma $\cdot$ sludge interaction, as sigma is taken from its low to high levels, the \% $\mathrm{U}$ removed tends to decrease slightly when sludge is present (Sludge = "Y") but it tends to decrease even more dramatically when sludge is not present. This particular interaction term, although significant in the statistical model, is also difficult to physically interpret.

\section{The Role of Al and Si Stoichiometry on Overall Uranium Removal}

The experimental program presented in this report also contained a series of eight runs as previously shown in Table 3. These runs, which are shown in Table 5, contained the same absolute product of aluminum and silicon concentration as in the 1:1 case. However, instead of the 1:1 stoichiometry used in the first 24 runs of the primary design these 8 runs consisted of half at 5:1 aluminum/silicon and the other half at 1:5 aluminum/silicon. 


$\begin{array}{cccccc}\text { Sigma } & \text { Conc } & \text { Temp } & \text { Time } & \begin{array}{c}\text { Sludge } \\ \text { Present }\end{array} & \begin{array}{c}\text { Removed } \\ 0\end{array} \\ 0 & 0 & 0 & \mathrm{Y} & 23 \\ 0 & 0 & 0 & 0 & \mathrm{Y} & 29 \\ 0 & 0 & 0 & 0 & \mathrm{Y} & 21 \\ 0 & 0 & 0 & 0 & \mathrm{Y} & 24 \\ 0 & 0 & 0 & 0 & \mathrm{~N} & 29 \\ 0 & 0 & 0 & 0 & \mathrm{~N} & 32 \\ 0 & 0 & 0 & 0 & \mathrm{~N} & 23 \\ 0 & 0 & 0 & 0 & \mathrm{~N} & 21\end{array}$

\section{Table $5 \%$ U Removed for Eight Stoichiometric Runs (in Coded Factor Levels)}

The four runs performed at the aluminum/silicon ratio of 1:1 had an average final uranium in solution of $17.1 \mathrm{ppm}(15.8,17.6,16.5$, and $18.3 \mathrm{ppm})$ with a $95 \%$ uncertainty in the mean of $\pm 1.6 \mathrm{ppm}$ when averaged over all conditions. The series of runs at the ratio of 5:1 aluminum/silicon had an average final uranium in solution of $16.4 \mathrm{ppm}(17.6,16.3,16.3$, and $15.4 \mathrm{ppm})$ with a $95 \%$ uncertainty in the mean of $\pm 2.0 \mathrm{ppm}$. The series of runs at the ratio of $1: 5$ aluminum $/ \mathrm{silicon}$ had a final average uranium in solution of $17.8 \mathrm{ppm}(18.0,17.4,17.7$, and $18.0 \mathrm{ppm})$ with a $95 \%$ uncertainty of $0.6 \mathrm{ppm}$.

The confidence intervals of all three of these conditions of stoichiometry overlap thus we interpret at the associated levels of statistical confidence that stoichiometry for the conditions evaluated is not significant. It is important to note that the previously defined threshold in the aliquot-based analysis of $17.5 \mathrm{ppm}$ is the boundary that allows for a statistical inference of uranium removed from solution. The 1:1 and 5:1 stoichiometry cases both have at least two of their four data points less than this value. Thus we can conclude at $95 \%$ confidence that uranium is removed in these tests. This is further reinforced by their respective means of 17.1 and $16.4 \mathrm{ppm}$. However, only one of the 1:5 stoichiometry data set is less than $17.5 \mathrm{ppm}$ and the mean is 17.8 , well above the $17.5 \mathrm{ppm}$ level required for statistical inference of uranium being removed from solution. Thus, there is evidence that the 1:5 and 5:1 data demonstrate different propensities to remove uranium from solution. Furthermore, there is evidence that neither the 1:5 nor the 5:1 data demonstrate different propensities to remove uranium relative to the intermediate 1:1 stoichiometric data. Further experimental work 
Rosencrance, et al.

WSRC-TR-2002-00232

Page 26 of 51

would be necessary to delineate the role, if any, in this range of experimental factors of stoichiometry on uranium removal from solution.

\section{The Overall Effect of Supersaturation with Respect to Al and/or Si on Uranium Removal}

As discussed earlier, one goal of these tests is to consider the possibility of an accelerated removal of uranium to the solid phase as a result of an efficient interaction of uranium with various aluminosilicate precursor phases. Specifically, it has been suggested that a higher fraction of uranium was retained in the accumulated mass of the fouled $2 \mathrm{H}$-evaporator relative to retention of $\mathrm{Si}$ and $\mathrm{Al}$ species. As a result of this suggestion it has been postulated that the primary mechanism for uranium removal in the fouled $2 \mathrm{H}$ evaporator could have been a scrubbing of uranium via aluminosilicate precursors. The following discussion addresses this potential for this concept following evaluation of our data set.

The previous discussion with respect to the effect of sigma on uranium removal focussed on the average effect of sigma on uranium removal as shown in Figure 3. Clearly the effect on average with increasing sigma is that the uranium removal decreases.

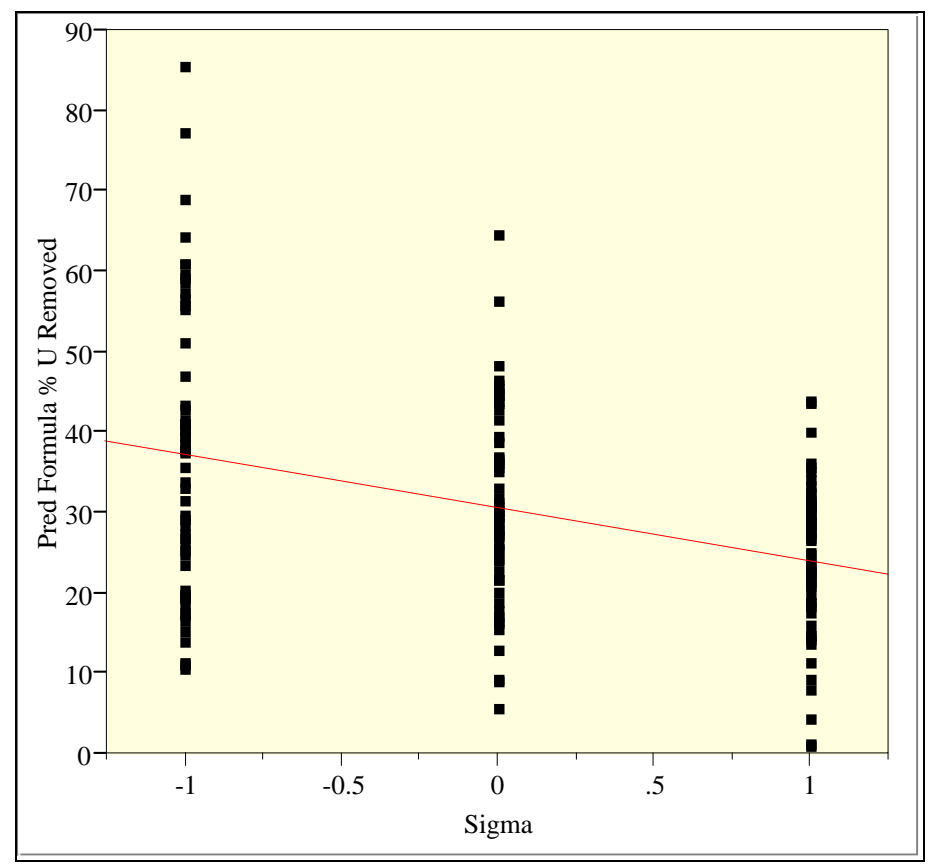

Figure 3 Average Effect of Sigma on \%U Removed 
Rosencrance, et al.

WSRC-TR-2002-00232

Page 27 of 51

It is important to note that certain combinations of other factor levels can lead to regions of the parameter space wherein the net effect of sigma on uranium removal is positive. This is a direct result of the model possessing multiple factors and a variety of sigma containing interaction terms. The experimental factors other than sigma in the model were combined at each of the possible 54 $\left(3^{3} \times 2\right)$ possible combinations of parameter space. Each of these 54 sets of conditions is then plotted as a function of increasing sigma and shown in Figure 4.

Figure 4 contains all of these 54 combinations of parameter space as functions of sigma. 12 of these lines demonstrate increasing uranium removal with increasing levels of sigma (supersaturation with respect to $\mathrm{Al}$ and $\mathrm{Si}$ ). The majority of the conditions, the other 42 lines, demonstrate the opposite effect. Each of these 12 sets of conditions includes sludge. Furthermore, the tendency to demonstrate this net overall effect of sigma for increased uranium removal is most favorable at lower concentration factors. The preference of this effect with regard to time and temperature are less pronounced and more evenly distributed. The 12 combinations of parameter levels that lead to this net positive effect of sigma on uranium removal are contained in appendix B.

In summary, when uranium removal is evaluated overall the average effect is such that removal decreases with increasing sigma. However, it is important to realize that the 42 combinations of conditions demonstrating decreased uranium removal with increased sigma dominate this average. More specifically, the 12 combinations of conditions that leads to the opposite effect are present but are overwhelmed and masked when averaged with the other 42 conditions. It is only when a more thorough evaluation is performed that the effect of sigma to increase uranium removal becomes apparent for this 12 condition subset of the parameter space. 
Rosencrance, et al.

WSRC-TR-2002-00232

Page 28 of 51

Finally, if one is to relate these results to deposition it is necessary to assume that the partition of the various uranium removal mechanisms is constant. Thus one would expect the deposition of uranium to correlate readily with the amount of uranium removed from solution. If indeed a significant alteration of the mechanistic partition for uranium removal is present then one must realize that although the ability to describe the amount of uranium removed from solution remains intact the assumption that the amount removed correlates with the amount deposited would no longer necessarily be reasonable.

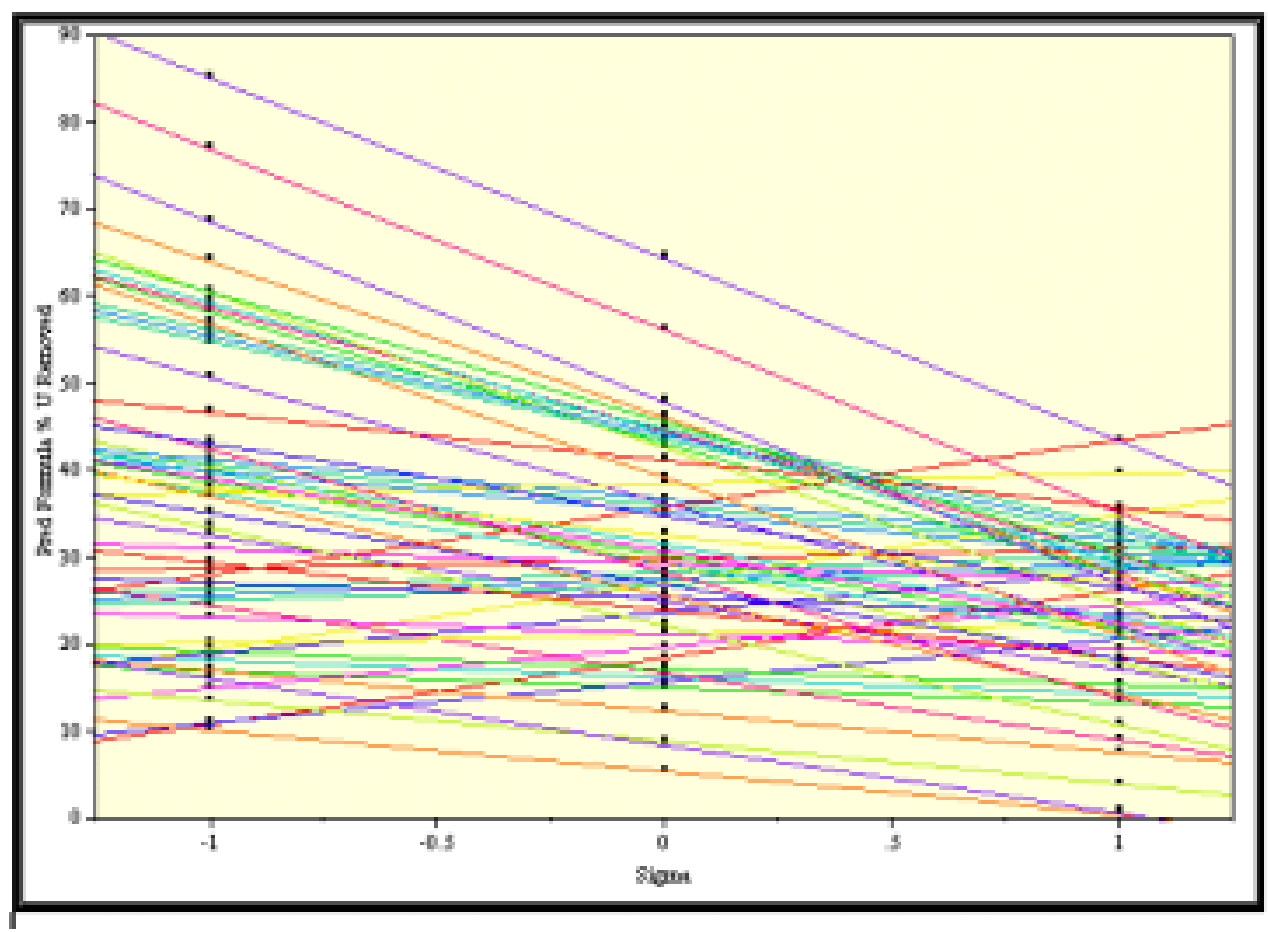

Figure 4 Effect of Individual Combinations of Factor Levels on \% U Removed

\section{Overall Application of Results to Plant Operation}

Uranium was removed from solutions in all solutions tested. This uranium removal has been hypothesized to be explained by a combination of 1.) precipitation of uranium containing phases from the matrix that was initially supersaturated with respect to uranium and 2.) in some cases for limited ranges of sigma (supersaturation with respect to $\mathrm{Si}$ and $\mathrm{Al}$ ) increased uranium partition to the solid phase with increasing sigma. 
Rosencrance, et al.

WSRC-TR-2002-00232

Page 29 of 51

One extension in our interpretation would be that this evidence supports the concept, as postulated by Lahoda, that aluminosilicate precursors could scrub uranium from solution. Interestingly, these results reveal that this process of removal is not widespread for the conditions evaluated and is a localized phenomena that only dominates removal in a limited number of cases.

Furthermore, a concept has been introduced into our understanding or uranium removal in systems containing aluminate and silicate. Namely, it has been hypothesized that for the majority of conditions evaluated the effect of $\mathrm{Si}$ and $\mathrm{Al}$ on the activity and thus solubility of uranium could dominate the removal of uranium from solution. If this effect is indeed controlling the uranium removal this concept could serve to underpin our current understanding of uranium removal from solution. Namely, it has implications on any scenario where uranium precipitation is of pertinence.

Thus, for the range of conditions evaluated at the fixed level of uranium concentration (20 ppm) in this test one can say that uranium removal occurred when concentrates were mixed. This removal is hypothesized to be partitioned between the two previously discussed effects. The quantitative partition between these two processes depends on the matrix conditions but most of the time the overall effects is proposed to be dominated by the impact of aluminate and/or silicate on uranium solubility.

For similar concentrate matrices one would expect that increasing the uranium concentration would only accentuate the uranium removal trends observed at an initial uranium concentration of $20 \mathrm{ppm}$. The ability of the concentrates to remove uranium from solution at lower uranium concentrations was not examined in this scope. It would be expected that maintaining the concentrate matrix and lowering the initial uranium concentration would tend to reduce the tendency of the system to form solids. In order to quantitatively define this effect further evaluation is necessary.

\section{Physical Properties, SEM and XRD Results}

As discussed in the TTP governing this work the physical property evaluation is qualitative in nature. Accompanying this report is a $\mathrm{CD}$ that contains an interactive format for evaluation of the video 
Rosencrance, et al.

WSRC-TR-2002-00232

Page 30 of 51

from the individual experimental runs. This video allows for direct evaluation of the solids formed during each experiment and their response to agitation and mixing. In general, the cakes are most brittle at increased levels of sigma. It is important to note that for the conditions evaluated most all of these cakes were brittle and could be easily fragmented with agitation. One point of interest would be to evaluate the characteristics of these cakes subsequent to extended aging.

XRD was performed on the solids obtained in six of the 32 experiments. These six were composed of three groups of two each (each grouping contains one sample with sludge and one sample without sludge). The first grouping corresponds to a low sigma value (no supersaturation with respect to $\mathrm{Si}$ and $\mathrm{Al}$ ), the second group corresponds to a high sigma level, and the final grouping corresponds to an intermediate sigma level. It should also be noted that the third grouping of samples with an intermediate level of sigma also consisted of an Al/Si stoichiometry of 5:1 while the first two groupings of samples demonstrated the standard 1:1 stoichiometric relation between Si and Al. Finally, the two samples in the low sigma level grouping correspond to the highest uranium removal observed in this set of experiments both for sludge and no sludge conditions.

All samples with sigma at the intermediate and high levels of sigma demonstrate characteristic peaks of aluminosilicate solid phases. The two runs performed at the low level of sigma (not saturated with respect to aluminosilicate formation) do not contain the characteristic peaks of aluminosilicate. These observations are as expected. Furthermore, none of these samples demonstrated uranium containing crystalline patterns above the detection limit.

Likewise SEM was performed on the solids obtained in six of the 32 experiments. These results are shown in appendix D. The samples correspond to the previously discussed groupings utilized in the XRD evaluation. The SEM spectra were collected in both secondary electron (SE) mode and back scattered electron (BSE) mode. The images from the SE mode typically demonstrate better resolution of morphological features for aluminosilicate structures while the images collected from the BSE mode provide increased sensitivity for elements of higher atomic number such as uranium. 
Rosencrance, et al.

WSRC-TR-2002-00232

Page 31 of 51

The morphology observed for the high and intermediate sigma sample groupings is very similar to the characteristic spherical yarn balls associated with sodalite and cancrinite. Uranium was not observed in any of the images collected for these two groupings of samples. This likely is associated with the fact that at higher levels of sigma significantly more aluminosilicate solids form and thus the mass fraction of uranium present in the collected solids is small. Thus the probability of observing uranium when selecting a portion of solids for analysis is decreased relative to lower supersaturation cases.

The morphology observed for the low sigma sample grouping (no saturation with respect to aluminosilicate formation) is quite different than that observed for the previous two groupings at higher levels of sigma. The solids that formed were smooth and elongated relative to the structures observed for the higher sigma samples. This is not surprising since the system did not contain the necessary supersaturation with respect to $\mathrm{Si}$ and $\mathrm{Al}$ to produce aluminosilicate solids.

\section{Items for Future Consideration}

Some of the issues that have evolved during the course of this experimental program would require further work to more firmly derive impacts on application and operational strategy. For instance, all regions of parameter space that demonstrate a net positive effect of sigma on uranium removal included sludge. Understanding of the amount and type of solid phase on this effect could be valuable.

Another item of interest originates with our observation that uranium is removed from solution and under certain system conditions this removal can be increased with increasing sigma. A more thorough evaluation of the means of uranium incorporation and its interactions with the other solid phases such as aluminosilicates is of interest since a large portion of the uranium removed likely was diuranate and only was physically associating with the aluminosilicate matrix. A simple physical association does not necessarily suggest increased deposition on evaporator surfaces. On the other hand the possibility could exist that even through the overall amount of uranium removed from solution decreases that it could be in a more unfavorable form such that more deposition would occur 
Rosencrance, et al.

WSRC-TR-2002-00232

Page 32 of 51

on evaporator surfaces. Significant scope is currently underway to evaluate these highly complex interactions. Further exploration in this area is likely warranted.

Finally, the hypothesized effect of $\mathrm{Si}$ and $\mathrm{Al}$ on the solubility of uranium is of interest and could serve to underpin our current understanding of the net effect of sigma on uranium removal. Based on the data obtained during this experimental program it appears that uranium solubility could be dramatically impacted by the presence of $\mathrm{Al}$ and/or $\mathrm{Si}$ in solution. Furthermore, if indeed this effect exists this concept could potentially lead to a mitigation strategy for uranium incorporation in scale. Specifically, if similar inorganic species to aluminate or silicate could be added to systematically alter the activity coefficient and the solubility of uranium the desupersaturative driving force for uranium removal would be dampened. Of course, this would assume that mechanisms such as coprecipitation are contributory to the uranium observed in the evaporator scale.

From a slightly different perspective this concept could explain additional uranium precipitated during evaporator. Specifically, if the presence of supersaturation with respect to $\mathrm{Si}$ and/or $\mathrm{Al}$ facilitates a change in the activity of uranium so as to increase uranium solubility then it could also be expected that desupersaturation with respect to $\mathrm{Si}$ and/or $\mathrm{Al}$ would have the opposite effect on uranium activity and lower the solubility.

Having observed aluminosilicate solids in the evaporator it is clear that at some times the system has been desupersaturated with regard to $\mathrm{Si}$ and/or $\mathrm{Al}$. If indeed uranium solubility is dependant on $\mathrm{Si}$ and/or $\mathrm{Al}$ in solution then following formation of aluminosilicate the liquor could find itself supersaturated with regard to uranium and begin to approach equilibrium thus forming additional uranium solids. In summary, if the presence of $\mathrm{Si}$ and/or $\mathrm{Al}$ in solution was a means to mobilize more uranium to the liquid phase only to return it to the solid phase following desupersaturation with respect to $\mathrm{Si}$ and/or Al. This could potentially contribute to an explanation of uranium solids in the evaporator system following the onset of scale problems and thus the amount of uranium solids precipitated and available for deposition would also correspondingly change. 
Rosencrance, et al.

WSRC-TR-2002-00232

Page 33 of 51

A simple set of experiments evaluating equilibrium uranium solubility as a function of aluminate and silicate levels could verify the presence of this phenomenon. If indeed this concept is demonstrated a series of tests could be performed to identify soluble inorganic species that don't readily precipitate or form troublesome scale while simultaneously increasing the uranium activity and solubility and thus assist in mitigating criticality concerns in deposited aluminosilicates. This impact interrelates with the previous two areas for suggested work and collectively would provide a more thorough technical basis for the long-term viability of the segregation strategy. Of course a common thread throughout all future evaluation would be a continued look at the derived model and the ability of this model to explain future experimental results.

\section{Conclusion}

The current testing was performed in order to assist in defining technical issues that surround the segregation strategy. This testing is focussed on the time frame subsequent to when the previously segregated concentrates are mixed and allowed to interact. Issues that were evaluated in the work included the partition of uranium between solid and liquid phases. Specifically, this serves as an indicator for the amount of uranium precipitated as well as the effect of supersaturation (with respect to $\mathrm{Si}$ and $\mathrm{Al}$ ) on uranium. The removal observed in this work is a simulation of when enriched concentrates of silicon and aluminum that were formed during a segregational operational strategy either purposefully or inadvertently interact. The formed solids were also qualitatively evaluated for potentially problematic cementation/fusion characteristics.

The results were statistically evaluated to ensure the conclusions were valid. The statistical evaluation included development of a model. The resulting model contained a number of statistically significant factors and demonstrated no lack of fit. The primary factors in the model of statistical pertinence were sigma (supersaturation with respect to $\mathrm{Si}$ and $\mathrm{Al}$ ), time, and concentration factor of the solution. A number of interaction terms were identified in the model. 
Rosencrance, et al.

WSRC-TR-2002-00232

Page 34 of 51

The overall uranium removed from solution is proposed to be a combination of 1.) precipitation of uranium containing phases from the matrix that was initially supersaturated with respect to uranium and 2.) in some cases for limited ranges of sigma (supersaturation with respect to $\mathrm{Si}$ and $\mathrm{Al}$ ) a term that increases uranium partition to the solid phase. The physical properties of the resulting solids were somewhat hard and brittle but could be broken up into a slurry form by introduction of shear to the system.

A concept has been introduced into our understanding or uranium removal in systems containing aluminate and silicate. Namely, it has been hypothesized that for the majority of conditions evaluated the effect of $\mathrm{Si}$ and $\mathrm{Al}$ on the activity and thus solubility of uranium could dominate the removal of uranium from solution. If this effect is indeed controlling the uranium removal this concept could serve to underpin our current understanding of uranium removal from solution. Namely, it has implications on any scenario where uranium precipitation is of pertinence.

This work may also provide experimental evidence to support the concept, as postulated by Lahoda, that aluminosilicate precursors could scrub uranium from solution. Interestingly, these results reveal that this process of removal, if operative, is not widespread for the conditions evaluated and is a localized phenomena that only dominates removal in a limited number of cases.

Future work is underway to better understand the details of the uranium removal processes in liquor representative of evaporator conditions. In addition to the current work in this area a number of areas for further consideration have been discussed. These areas include 1.) Solids type and loading, 2.) A more thorough evaluation of uranium containing solid phases, 3.) An evaluation of the impact of $\mathrm{Si}$ and $\mathrm{Al}$ on the solubility of uranium, and finally 4.) Further consideration of a quantification of the physical properties of the resulting solids and their anticipated processability. 


\section{References}

${ }^{1}$ W. R. Wilmarth, S. D. Fink, D. T. Hobbs, and M. S. Hay, "Characterization and Dissolution Studies of Samples from the 242-16H Evaporator Gravity Drain Line (U)," WSRC-TR-97-0326, Rev. 0, October 16, 1997.

${ }^{2}$ W. R. Wilmarth, C. J. Coleman, J. C. Hart, and W. T. Boyce, "Characterization of Samples from the 242-16H Evaporator Wall," WSRC-TR-2000-00089, March 20, 2000.

${ }^{3}$ W. R. Wilmarth, D. D. Walker, and S. D. Fink, "Sodium Aluminosilicate Formation in Tank 43H Simulants," WSRC-TR-97-00389, Rev. 0, November 15, 1997.

${ }^{4}$ W. R. Wilmarth, V.H. Dukes, and J.T. Mills, "Task Technical and Quality Assurance Plan for Aluminosilicate Formation Testing," WSRC-RP-2000-01027, Rev. 0, December 15, 2000.

${ }^{5}$ S.W. Rosencrance, D. Herman, and D. Healy, "Task Technical and Quality Assurance Plan for Continuous Aluminosilicate Deposition Testing" WSRC-RP-2001-0731, Rev. 0, August 15, 2001. ${ }^{6}$ S.W. Rosencrance, D. Herman, and D. Healy, "Formation and Deposition of Aluminosilicates in Support of the 2H Evaporator Fouling Program" WSRC-TR-2001-0464, Rev. 0, October 2001.

${ }^{7}$ J. England, "High Si-Al-U Concentrate Interaction Testing", HLE-TTR-2002-011, Rev. 0, October 31, 2001.

${ }^{8}$ L. O Dworjanyn, S. C. Smith, A. L. Williams, “ Task Technical and Quality Assurance Plan for Evaporator Scaling Dynamics, WSRC-RP-2000-00963, Rev. 0, November 16, 2000.

${ }^{9}$ David Hobbs, private communication $11 / 15 / 01$.

${ }^{10}$ David Hobbs, private communication 11/15/01.

${ }^{11}$ W. R. Wilmarth, V. H. Dukes, J. T. Mills, T. B. Edwards, "Results of Aluminosilicate Formation Testing," WSRC-TR-2001-00330, July 13, 2001

${ }^{12}$ L. O Dworjanyn, S. C. Smith, A. L. Williams, “ Task Technical and Quality Assurance Plan for Evaporator Scaling Dynamics, WSRC-RP-2000-00963, Rev. 0, November 16, 2000.

13 "Design Verification and Checking," Manual E7, Procedure 2.40, Rev. 0, May 26, 1993.

14 Technical Notebook Use," Manual L1, Procedure 4.19, Rev. 5, February 2, 1998.

15 "Conduct of Research and Development," WSRC-IM-97-0024, Rev. 2, June 30, 2000.

${ }^{16}$ M. C. Barnes, J. Addai-Mensah, and A. R. Gerson, J. Cryst. Growth, 200 (1999), 251-264. 
Rosencrance, et al.

WSRC-TR-2002-00232

Page 36 of 51

\section{Appendix A Statistical Data}


Exhibit A1 Plots of \% U Removed versus the Experimental Factors

Plot of \% U Removed By Sigma

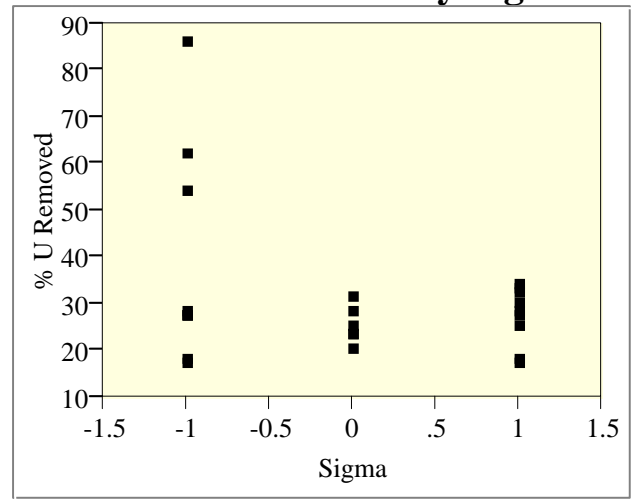

Plot of \% U Removed By Conc

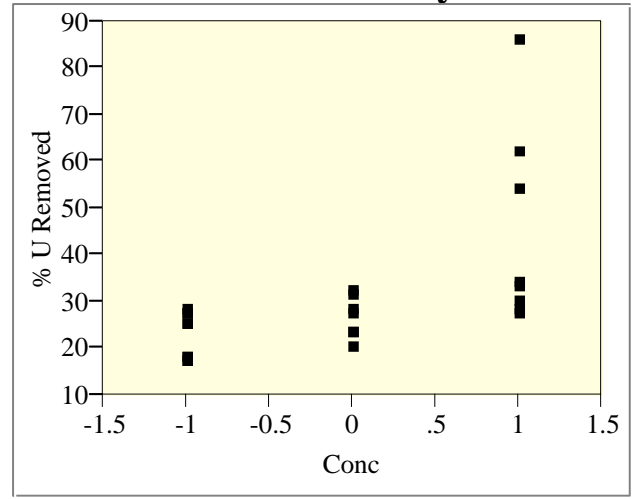

Plot of \% U Removed By Time

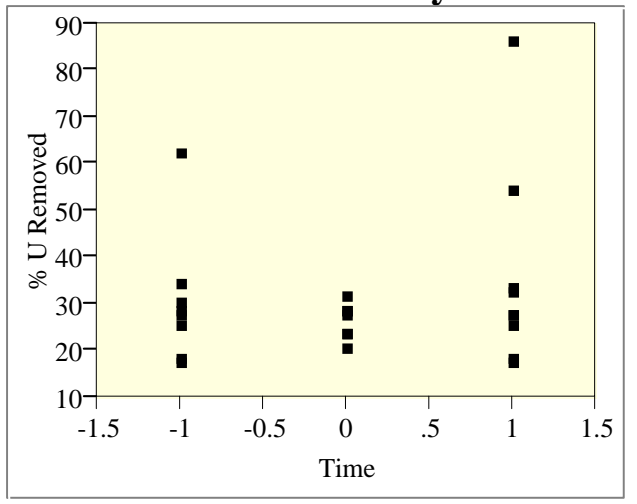

\section{Plot of \% U Removed By Sludge}

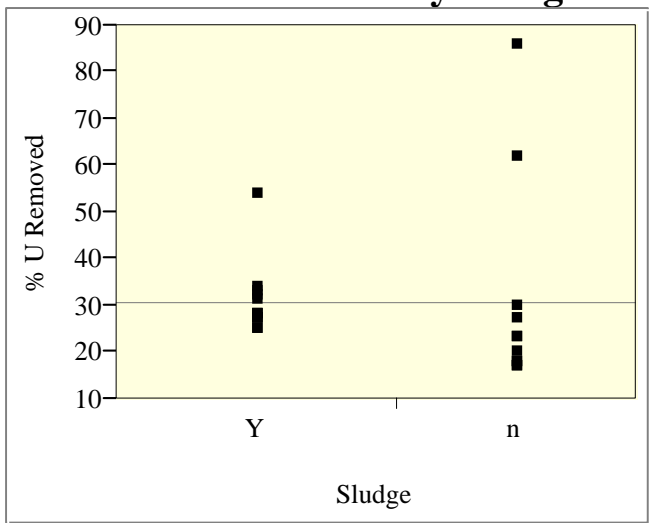

Plot of \% U Removed By Temp

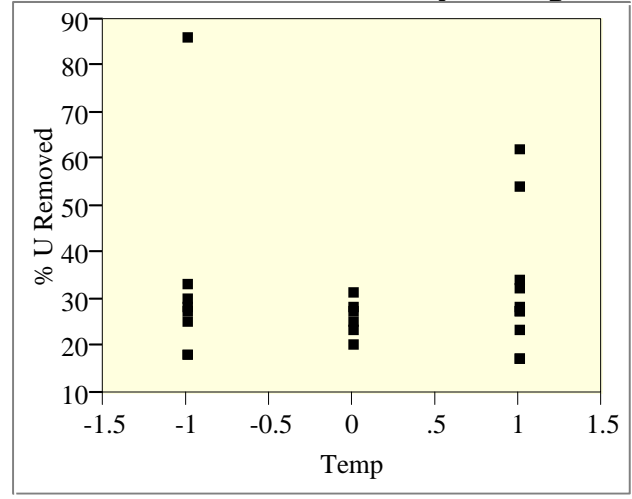




\section{Exhibit A2 Results of Fitting the Full Quadratic Model to the \% U Removed Data}

\section{Response \% U Removed \\ Actual by Predicted Plot}

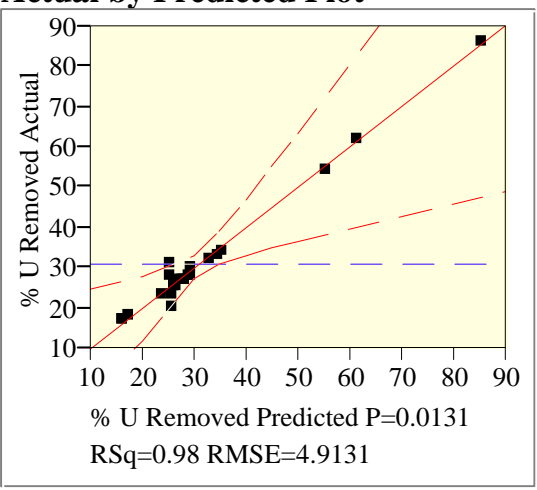

\section{Summary of Fit}

$\begin{array}{lr}\text { RSquare } & 0.982975 \\ \text { RSquare Adj } & 0.902105 \\ \text { Root Mean Square Error } & 4.913131 \\ \text { Mean of Response } & 30.83333 \\ \text { Observations (or Sum Wgts) } & 24\end{array}$

\section{Analysis of Variance}

$\begin{array}{lrrrr}\text { Source } & \text { DF } & \text { Sum of Squares } & \text { Mean Square } & \text { F Ratio } \\ \text { Model } & 19 & 5574.7779 & 293.409 & 12.1551 \\ \text { Error } & 4 & 96.5554 & 24.139 & \text { Prob }>\text { F } \\ \text { C. Total } & 23 & 5671.3333 & & 0.0131\end{array}$

\section{Lack Of Fit}

\section{Source}

Lack Of Fit

Pure Error DF

Total Error

2
2
4

2
2

Sum of Squares

87.555428
9.000000

96.555428

\section{Residual by Predicted Plot}

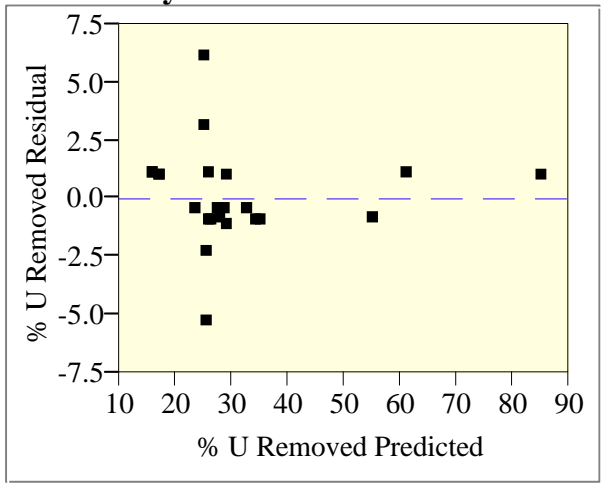

\section{Parameter Estimates}

$\begin{array}{lrrrr}\text { Term } & \text { Estimate } & \text { Std Error } & \text { t Ratio } & \text { Prob>|t| } \\ \text { Intercept } & 25.130931 & 2.195476 & 11.45 & 0.0003 \\ \text { Sigma } & -6.64912 & 1.189399 & -5.59 & 0.0050 \\ \text { Conc } & 11.084324 & 1.223237 & 9.06 & 0.0008 \\ \text { Sigma*Conc } & -6.602841 & 1.262208 & -5.23 & 0.0064 \\ \text { Temp } & 0.0406763 & 1.223237 & 0.03 & 0.9751 \\ \text { Sigma*Temp } & -0.054222 & 1.21584 & -0.04 & 0.9666 \\ \text { Conc*Temp } & -0.102841 & 1.262208 & -0.08 & 0.9390 \\ \text { Time } & 3.0406763 & 1.223237 & 2.49 & 0.0678 \\ \text { Sigma*Time } & -3.022159 & 1.262208 & -2.39 & 0.0748 \\ \text { Conc*Time } & 2.6772606 & 1.266117 & 2.11 & 0.1020 \\ \text { Temp*Time } & -4.772159 & 1.262208 & -3.78 & 0.0194 \\ \text { Sludge[Y] } & -0.193109 & 1.062674 & -0.18 & 0.8646 \\ \text { Sigma*Sludge[Y] } & 4.7258804 & 1.189399 & 3.97 & 0.0165 \\ \text { Conc*Sludge[Y] } & -5.790676 & 1.223237 & -4.73 & 0.0091 \\ \text { Temp*Sludge[Y] } & 3.7258804 & 1.189399 & 3.13 & 0.0351 \\ \text { Time*Sludge[Y] } & 0.4156763 & 1.223237 & 0.34 & 0.7511 \\ \text { Sigma*Sigma } & 0.6205721 & 4.28647 & 0.14 & 0.8919 \\ \text { Conc*Conc } & -2.089312 & 4.362525 & -0.48 & 0.6570 \\ \text { Temp*Temp } & 1.8427965 & 4.475991 & 0.41 & 0.7017 \\ \text { Time*Time } & 7.6606883 & 4.362525 & 1.76 & 0.1539\end{array}$




\section{Exhibit A2 Results of Fitting the Full Model to the \% U Removed Data (continued)}

\begin{tabular}{|c|c|c|c|c|c|c|c|c|c|c|c|c|c|c|c|c|c|c|c|c|}
\hline & & & & Sigma * & & Sigma* & Conc * & & Sigma * & Conc * & Temp * & & Sigma * & Conc * & Temp * & Time * & Sigma * & Conc * & Temp * & Time * \\
\hline & Intercept & Sigma & Conc & Conc & Temp & Temp & Temp & Time & Time & Time & Time & Sludge[Y] & Sludge $[\mathrm{Y}]$ & Sludge [Y] & Sludge $[\mathrm{Y}]$ & Sludge [Y] & Sigma & Conc & Temp & Time \\
\hline Intercept & 1.000 & 0.037 & 0.087 & 0.035 & -0.087 & -0.026 & 0.035 & -0.087 & -0.035 & 0.095 & -0.035 & -0.071 & 0.037 & 0.087 & 0.037 & -0.087 & -0.192 & -0.065 & -0.203 & -0.065 \\
\hline Sigma & 0.037 & 1.000 & 0.005 & -0.008 & -0.005 & -0.035 & -0.008 & -0.005 & 0.008 & -0.036 & 0.008 & -0.044 & -0.066 & 0.005 & -0.066 & -0.005 & 0.115 & 0.023 & -0.175 & 0.023 \\
\hline Conc & 0.087 & 0.005 & 1.000 & 0.028 & 0.008 & 0.007 & 0.028 & 0.008 & -0.028 & 0.027 & -0.028 & 0.041 & 0.005 & -0.008 & 0.005 & 0.008 & -0.059 & 0.171 & -0.041 & -0.112 \\
\hline Sigma*Conc & 0.035 & -0.008 & 0.028 & 1.000 & -0.028 & -0.018 & 0.053 & -0.028 & -0.053 & 0.063 & -0.053 & 0.008 & -0.008 & 0.028 & -0.008 & -0.028 & 0.024 & 0.093 & 0.037 & -0.181 \\
\hline Temp & -0.087 & -0.005 & 0.008 & -0.028 & 1.000 & -0.007 & -0.028 & -0.008 & 0.028 & -0.027 & 0.028 & -0.041 & -0.005 & 0.008 & -0.005 & -0.008 & 0.059 & 0.112 & -0.235 & 0.112 \\
\hline Sigma*Temp & -0.026 & -0.035 & 0.007 & -0.018 & -0.007 & 1.000 & -0.018 & -0.007 & 0.018 & -0.031 & 0.018 & -0.042 & -0.035 & 0.007 & -0.035 & -0.007 & -0.059 & 0.068 & -0.065 & 0.068 \\
\hline Conc*Temp & 0.035 & -0.008 & 0.028 & 0.053 & -0.028 & -0.018 & 1.000 & -0.028 & -0.053 & 0.063 & -0.053 & 0.008 & -0.008 & 0.028 & -0.008 & -0.028 & 0.024 & 0.093 & 0.037 & -0.181 \\
\hline Time & -0.087 & -0.005 & 0.008 & -0.028 & -0.008 & -0.007 & -0.028 & 1.000 & 0.028 & -0.027 & 0.028 & -0.041 & -0.005 & 0.008 & -0.005 & -0.008 & 0.059 & 0.112 & 0.041 & -0.171 \\
\hline Sigma*Time & -0.035 & 0.008 & -0.028 & -0.053 & 0.028 & 0.018 & -0.053 & 0.028 & 1.000 & -0.063 & 0.053 & -0.008 & 0.008 & -0.028 & 0.008 & 0.028 & -0.024 & 0.181 & -0.037 & -0.093 \\
\hline Conc*Time & 0.095 & -0.036 & 0.027 & 0.063 & -0.027 & -0.031 & 0.063 & -0.027 & -0.063 & 1.000 & -0.063 & 0.007 & -0.036 & 0.027 & -0.036 & -0.027 & -0.089 & 0.050 & -0.065 & 0.050 \\
\hline Temp*Time & -0.035 & 0.008 & -0.028 & -0.053 & 0.028 & 0.018 & -0.053 & 0.028 & 0.053 & -0.063 & 1.000 & -0.008 & 0.008 & -0.028 & 0.008 & 0.028 & -0.024 & 0.181 & -0.037 & -0.093 \\
\hline Sludge $[\mathrm{Y}]$ & -0.071 & -0.044 & 0.041 & 0.008 & -0.041 & -0.042 & 0.008 & -0.041 & -0.008 & 0.007 & -0.008 & 1.000 & -0.044 & 0.041 & -0.044 & -0.041 & -0.241 & -0.006 & 0.267 & -0.006 \\
\hline Sigma*Sludge $[\mathrm{Y}]$ & 0.037 & -0.066 & 0.005 & -0.008 & -0.005 & -0.035 & -0.008 & -0.005 & 0.008 & -0.036 & 0.008 & -0.044 & 1.000 & 0.005 & -0.066 & -0.005 & 0.115 & 0.023 & -0.175 & 0.023 \\
\hline Conc*Sludge $[\mathrm{Y}]$ & 0.087 & 0.005 & -0.008 & 0.028 & 0.008 & 0.007 & 0.028 & 0.008 & -0.028 & 0.027 & -0.028 & 0.041 & & 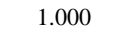 & 0.005 & 0.008 & -0.059 & 0.171 & -0.041 & -0.112 \\
\hline Temp*Sludge $[\mathrm{Y}]$ & 0.037 & -0.066 & 0.005 & -0.008 & -0.005 & -0.035 & -0.008 & -0.005 & 0.008 & -0.036 & 0.008 & -0.044 & -0.066 & 0.0 & 1.000 & -0.005 & -0.181 & 0.023 & 0.109 & 0.023 \\
\hline Time*Sludge[Y] & -0.087 & -0.005 & 0.008 & -0.028 & -0.008 & -0.007 & -0.028 & -0.008 & 0.028 & -0.027 & 0.028 & -0.041 & -0.005 & 0.008 & -0.005 & 1.000 & 0.059 & 0.112 & 0.041 & -0.171 \\
\hline Sigma*Sigma & -0.192 & 0.115 & -0.059 & 0.024 & 0.059 & -0.059 & 0.024 & 0.059 & -0.024 & -0.089 & -0.024 & -0.241 & 0.115 & -0.059 & -0.181 & 0.059 & 1.000 & -0.157 & -0.540 & -0.157 \\
\hline Conc*Conc & -0.065 & 0.023 & 0.171 & 0.093 & 0.112 & 0.068 & 0.093 & 0.112 & 0.181 & 0.050 & 0.181 & -0.006 & 0.1 & 0.171 & 0.023 & 0.112 & -0.157 & 1.000 & -0.191 & -0.585 \\
\hline Temp*Temp & -0.203 & -0.175 & -0.041 & 0.037 & -0.235 & -0.065 & 0.037 & 0.041 & -0.037 & -0.065 & -0.037 & 0.267 & -0.175 & -0.041 & 0.109 & 0.041 & -0.540 & -0.191 & 1.000 & -0.191 \\
\hline Time*Time & -0.065 & 0.023 & -0.112 & -0.181 & 0.112 & 0.068 & -0.181 & -0.171 & -0.093 & 0.050 & -0.093 & -0.006 & 0.023 & -0.112 & 0.023 & -0.171 & -0.157 & -0.585 & -0.191 & 1.000 \\
\hline
\end{tabular}


Exhibit A3 Results of Fitting the Reduced Model to the \% U Removed Data

\section{Response \% U Removed \\ Whole Model \\ Actual by Predicted Plot}

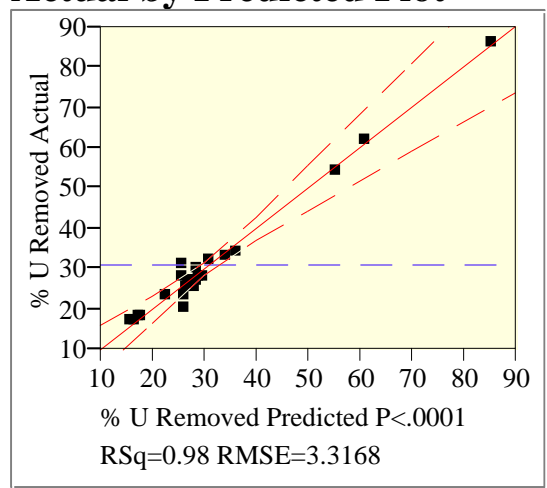

\section{Summary of Fit}

RSquare

RSquare Adj

0.980602

0.955385

Root Mean Square Error $\quad 3.316802$

Mean of Response $\quad 30.83333$

Observations (or Sum Wgts) 24

\section{Analysis of Variance}

Source DF Sum of Squares

Model $\quad 13 \quad 5561.3216$

$\begin{array}{lll}\text { Error } & 10 & 110.0117\end{array}$

C. Total $23 \quad 5671.3333$

Mean Square F Ratio $427.794 \quad 38.8862$

11.001 Prob $>$ F

\section{Lack Of Fit}

Source

Lack Of Fit

DF

Pure Error

8

Sum of Squares 101.01173
9.00000

9.00000
110.01173

Mean Square $<.0001$

12.6265

4.5000

F Ratio

2.8059

Prob > F

0.2892

Max RSq

0.9984

\section{Parameter Estimates}

Term

Intercept

Sigma

Conc

Sigma*Conc

Temp

Time

Sigma*Time

Conc*Time

Temp*Time

Sludge[Y]

Sigma*Sludge[Y]

Conc*Sludge[Y]

Temp*Sludge[Y]

Time*Time

$\begin{array}{rrrr}\text { Estimate } & \text { Std Error } & \text { t Ratio } & \text { Prob }>|t| \\ 25.538434 & 1.294626 & 19.73 & <.0001 \\ -6.575778 & 0.789546 & -8.33 & <.0001 \\ 11.209566 & 0.812571 & 13.80 & <.0001 \\ -6.579104 & 0.841809 & -7.82 & <.0001 \\ 0.2165465 & 0.798469 & 0.27 & 0.7918 \\ 3.0384003 & 0.808091 & 3.76 & 0.0037 \\ -2.92293 & 0.8359 & -3.50 & 0.0058 \\ 2.8018503 & 0.839874 & 3.34 & 0.0075 \\ -4.67293 & 0.8359 & -5.59 & 0.0002 \\ -0.237101 & 0.685163 & -0.35 & 0.7365 \\ 4.7992221 & 0.789546 & 6.08 & 0.0001 \\ -5.665434 & 0.812571 & -6.97 & <.0001 \\ 3.7471615 & 0.788861 & 4.75 & 0.0008 \\ 7.6249664 & 1.527814 & 4.99 & 0.0005\end{array}$

Residual by Predicted Plot

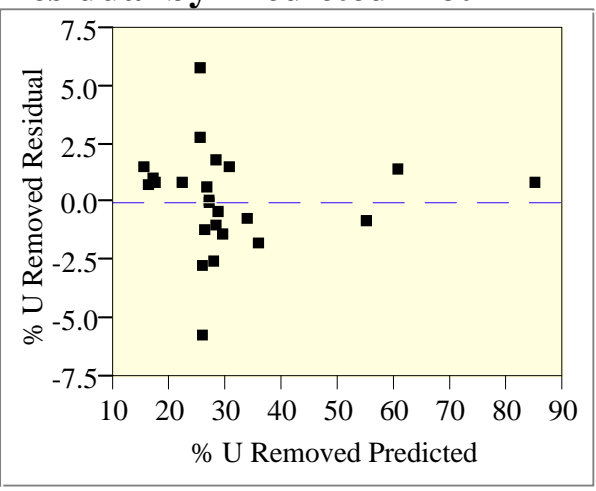



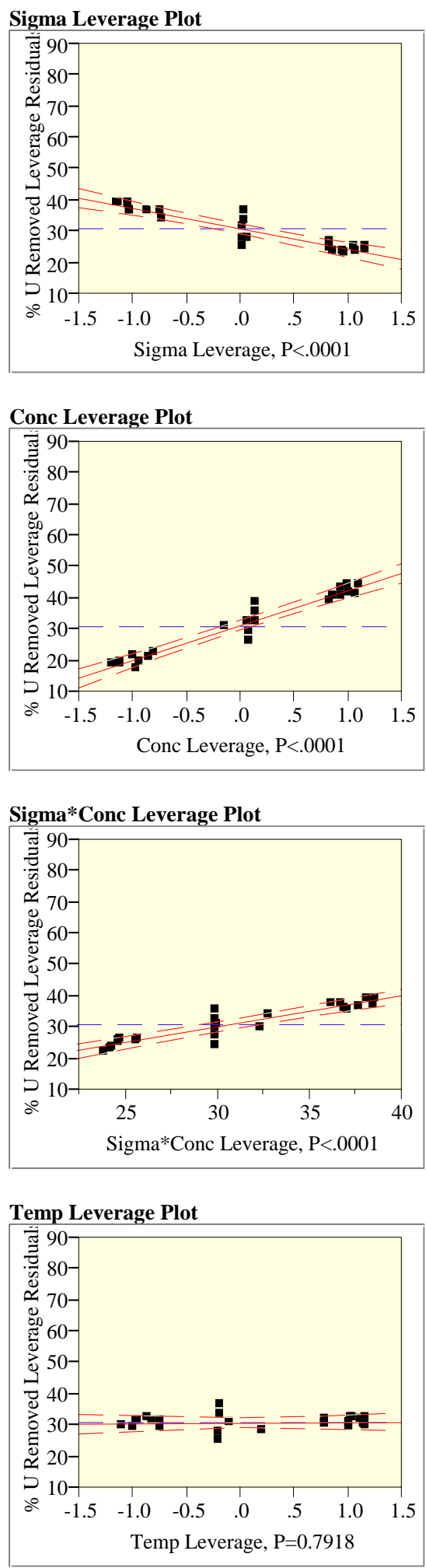
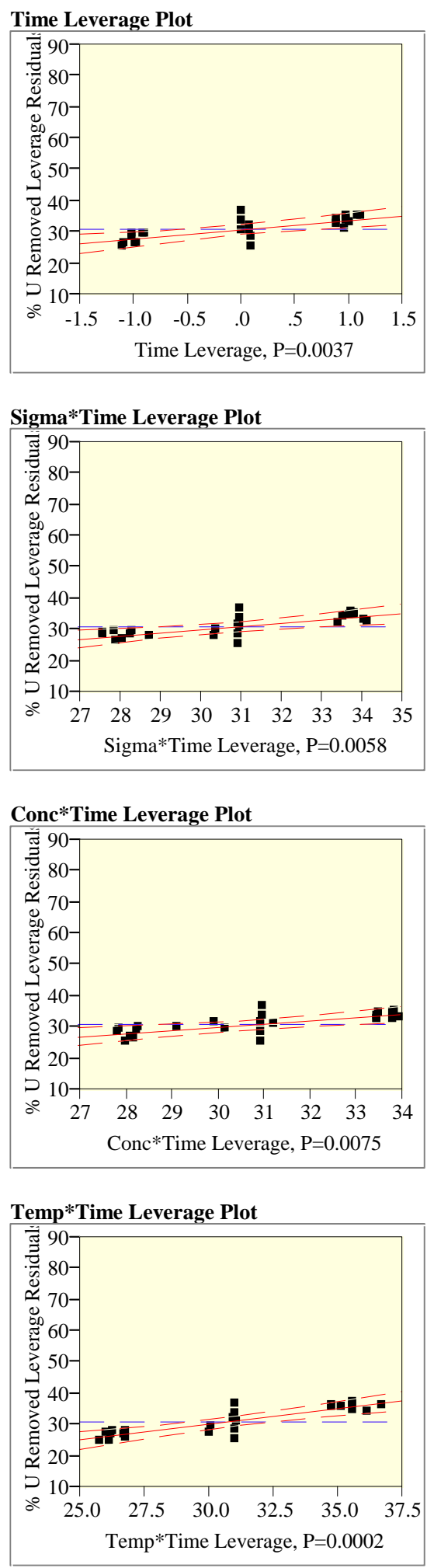

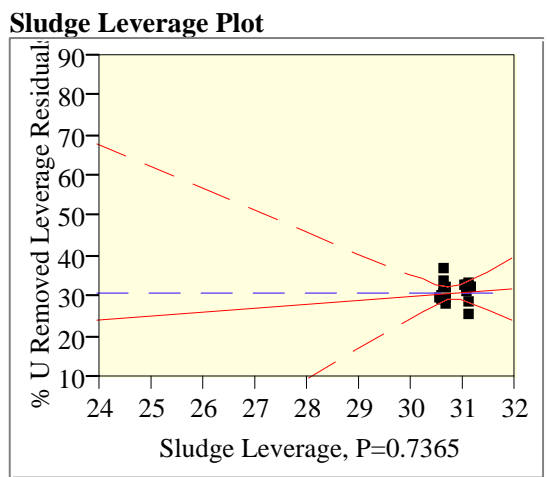

\section{Least Squares Means Table \\ Level Least Sq Mean Std Error Mean \\ $\begin{array}{llll}\text { Y } & 25.519384 & 1.4027063 & 30.6923\end{array}$}

Sigma*Sludge Leverage Plot

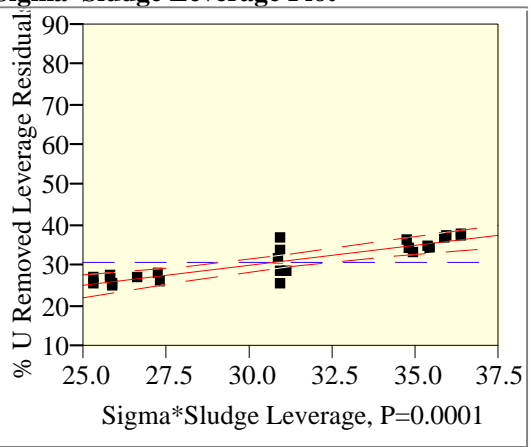




\section{Appendix B Factor Combinations leading to Positive Effect of Sigma Terms} on Uranium Removal 


\begin{tabular}{|c|c|c|c|c|}
\hline Condition & Conc. Factor & Temperature & Time & Sludge \\
\hline 1 & -1 & -1 & -1 & Y \\
\hline 2 & -1 & -1 & 0 & Y \\
\hline 3 & -1 & -1 & 1 & Y \\
\hline 4 & -1 & 0 & -1 & Y \\
\hline 5 & -1 & 0 & 0 & Y \\
\hline 6 & -1 & 0 & 1 & Y \\
\hline 7 & -1 & 1 & -1 & Y \\
\hline 8 & -1 & 1 & 0 & $\mathbf{Y}$ \\
\hline 9 & -1 & 1 & 1 & $\mathbf{Y}$ \\
\hline 10 & 0 & -1 & -1 & $\mathbf{Y}$ \\
\hline 11 & 0 & 0 & -1 & $\mathbf{Y}$ \\
\hline 12 & 0 & 1 & & -1 \\
\hline
\end{tabular}


Rosencrance, et al.

WSRC-TR-2002-00232

Page 44 of 51

\section{Appendix C SEM Images}



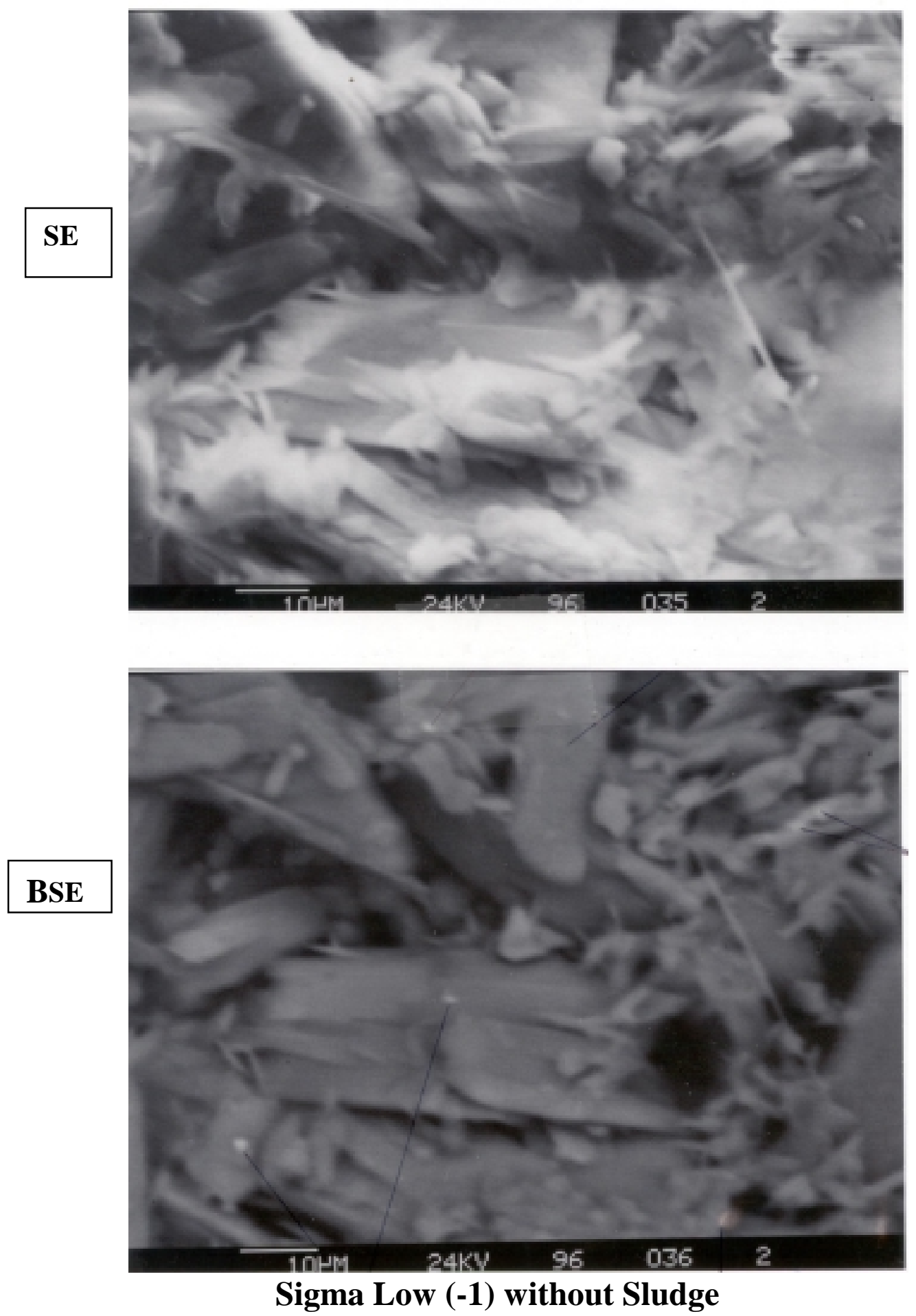

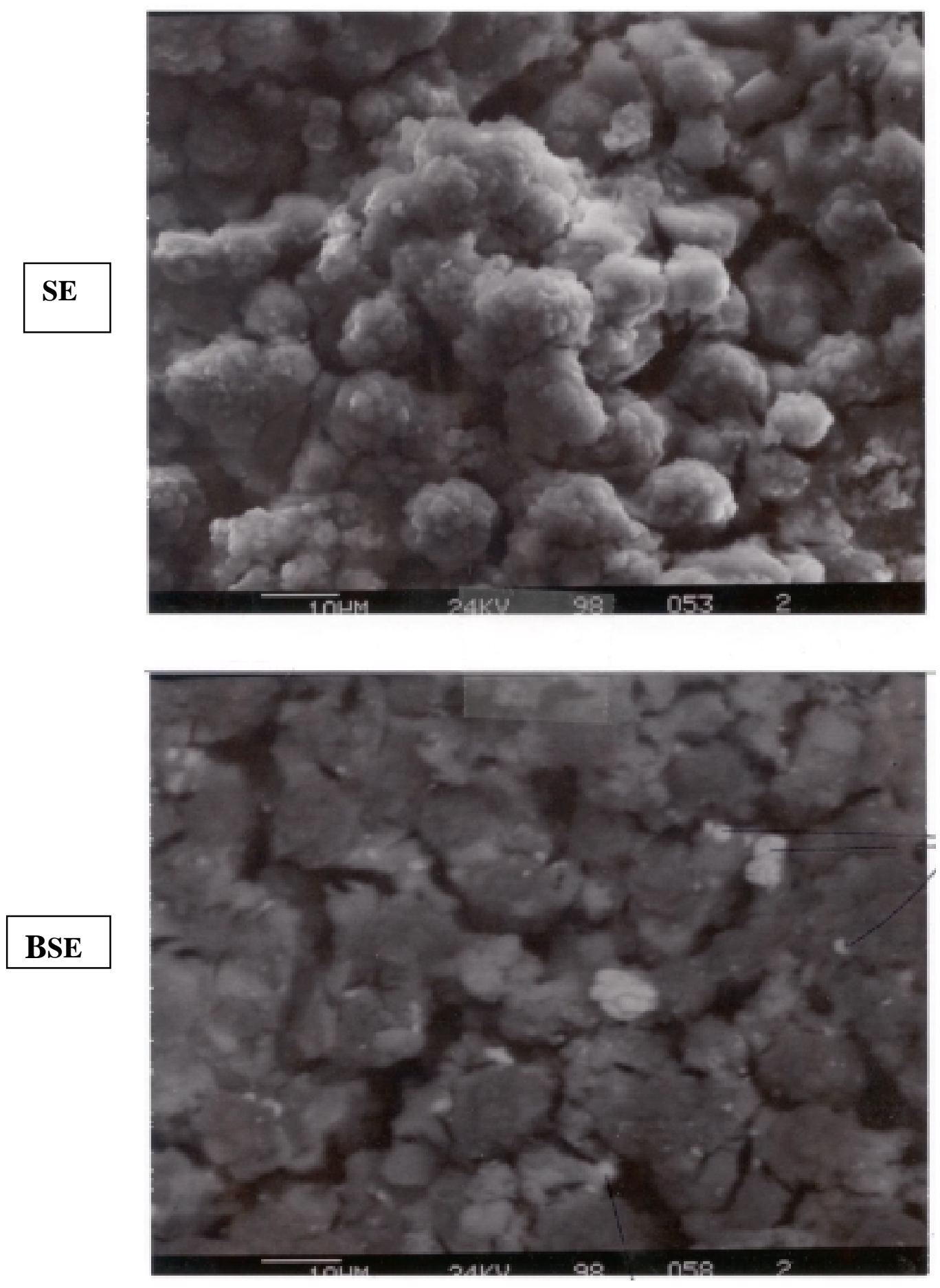

Sigma Intermediate (0) without Sludge 

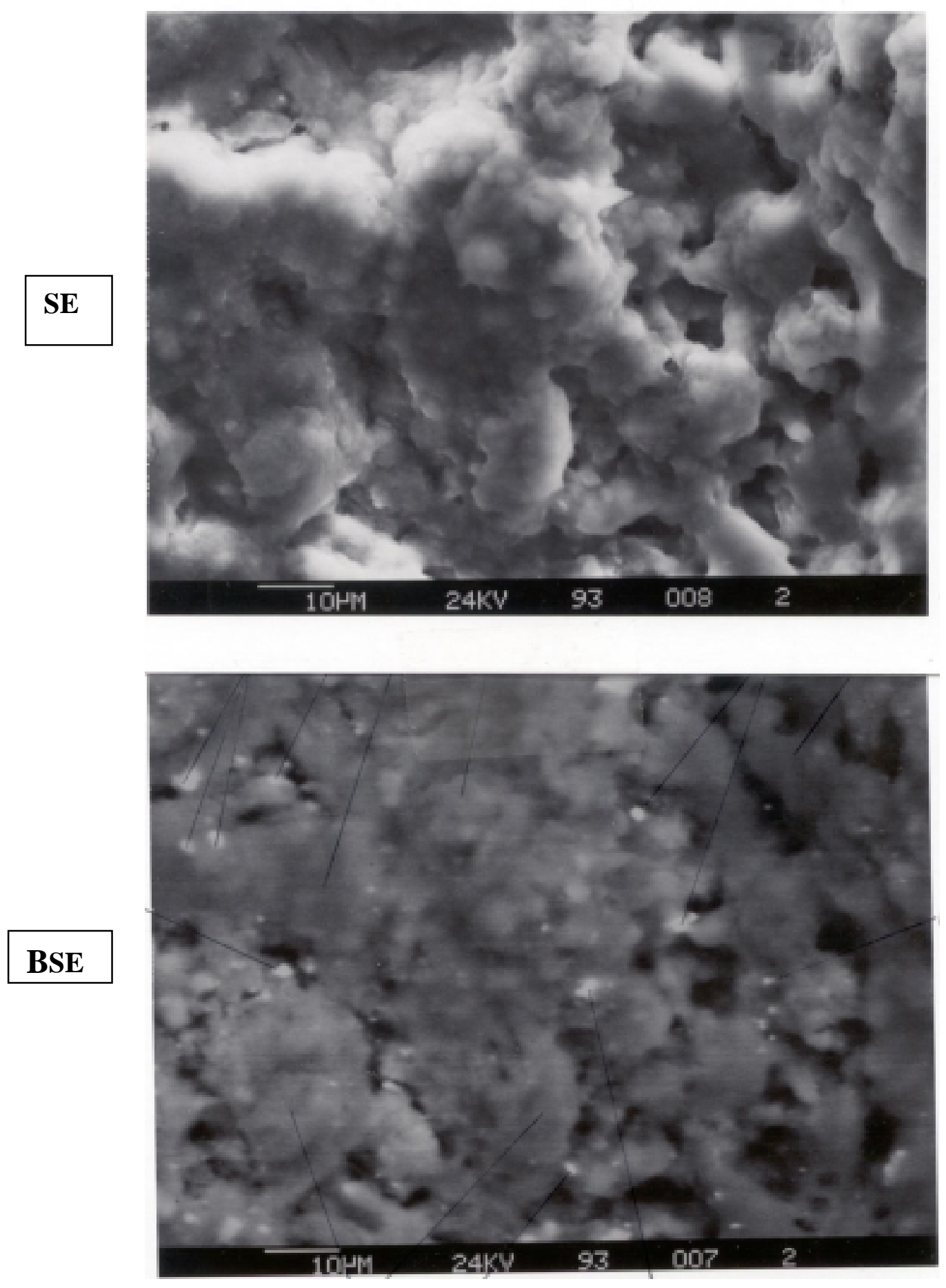

Sigma High (1) without Sludge 

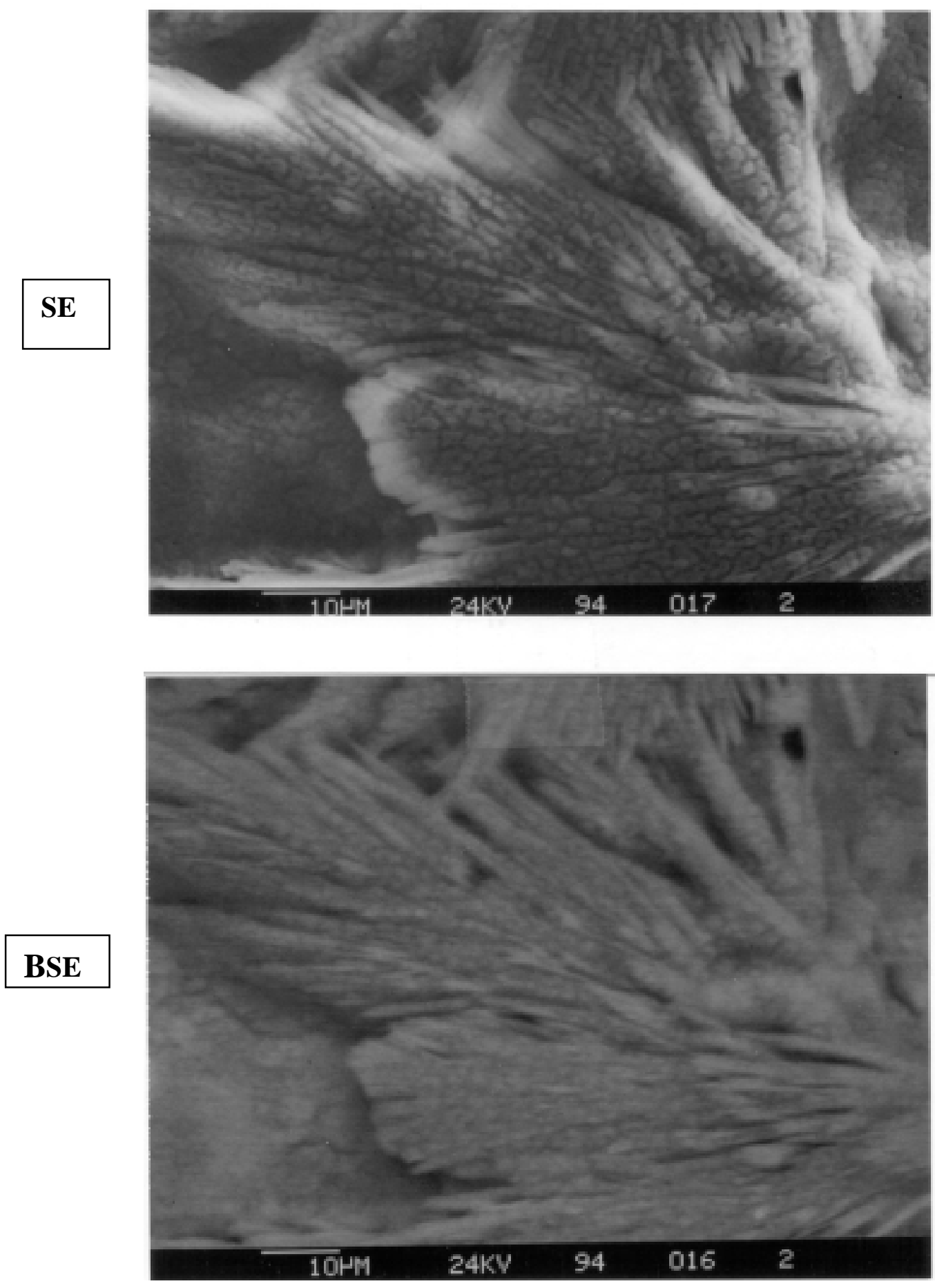

Sigma Low (-1) with Sludge 

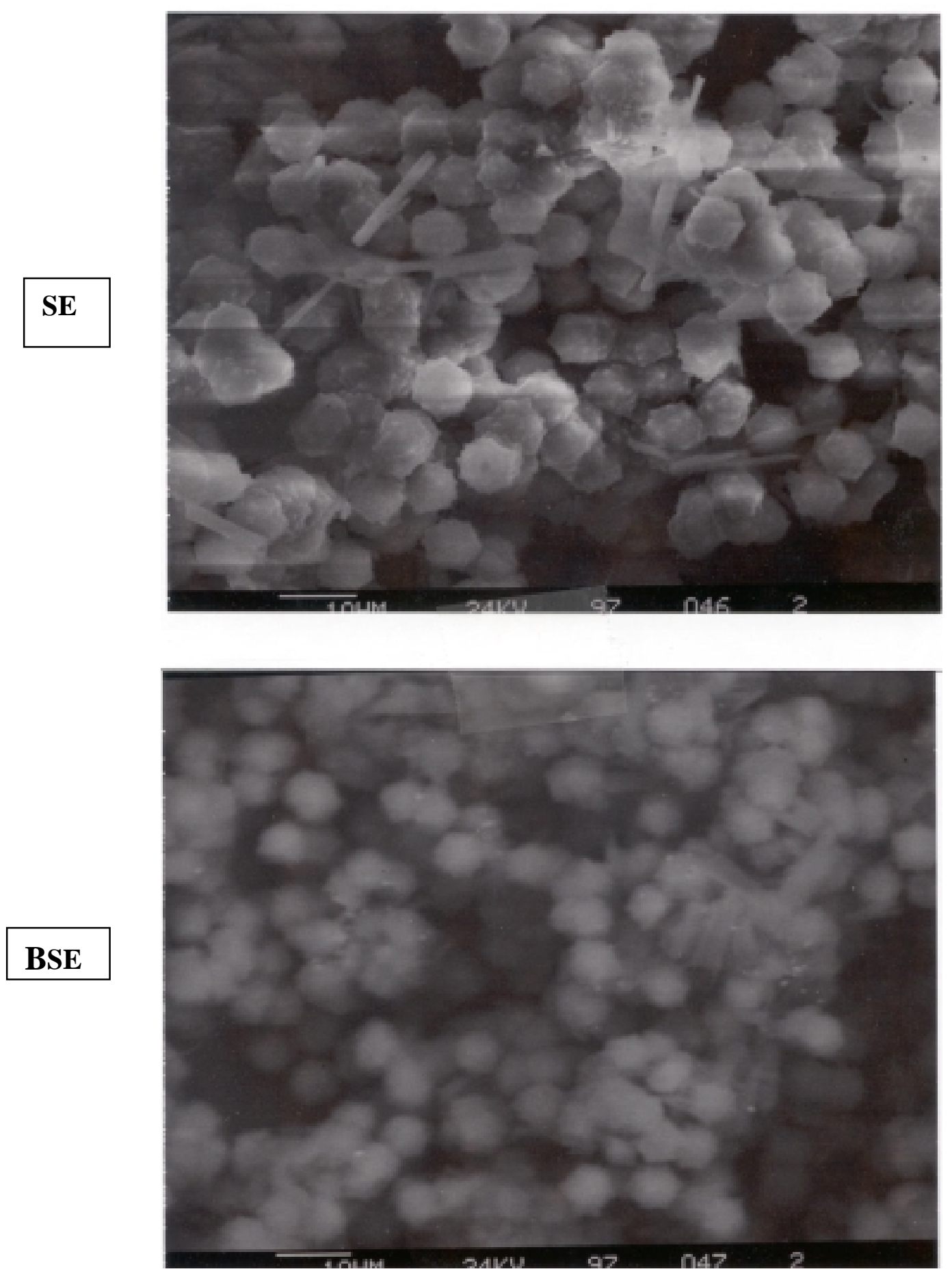

Sigma Intermediate (0) with Sludge 

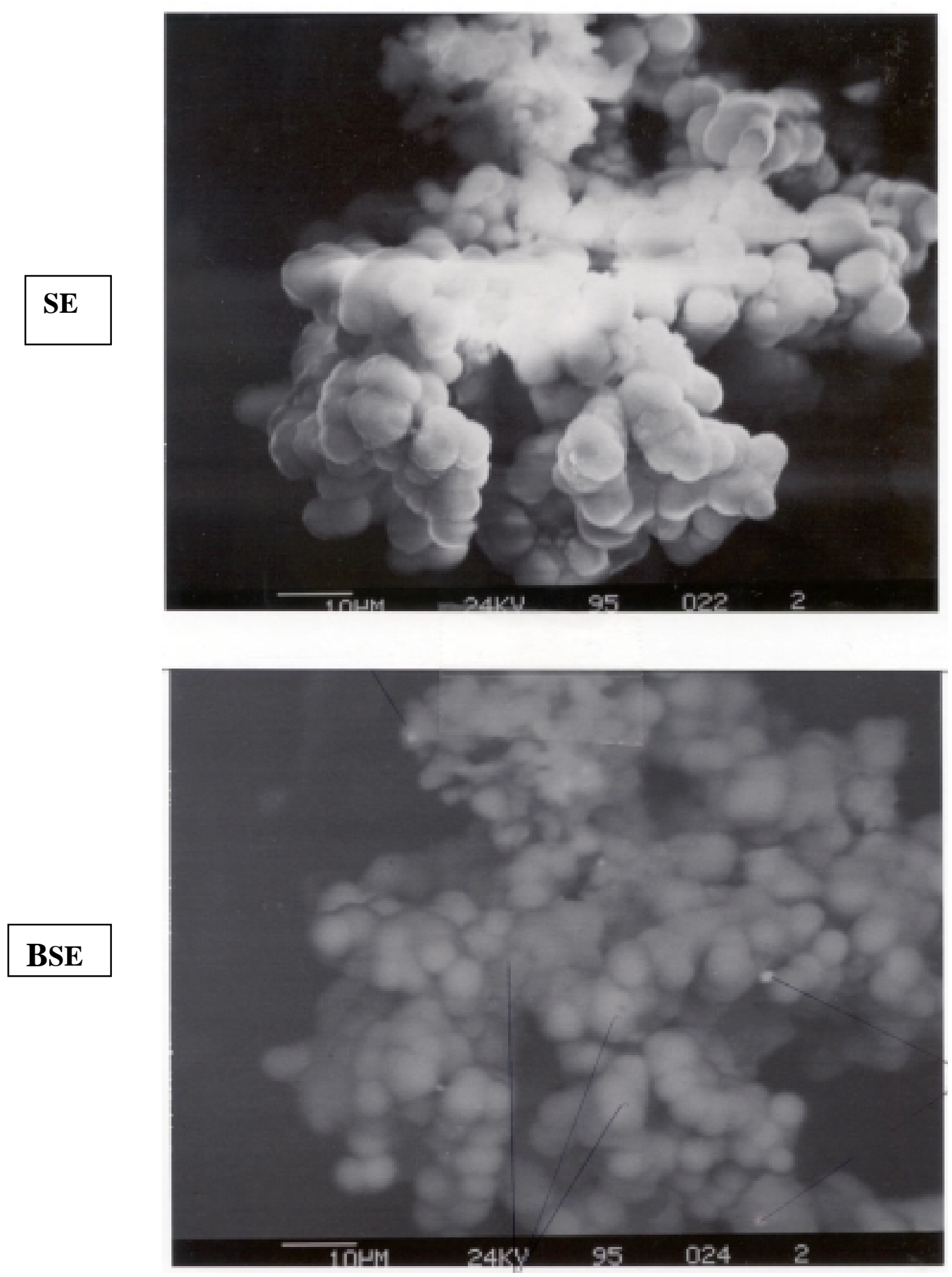

Sigma High (1) with Sludge 
This page is intentionally left blank 